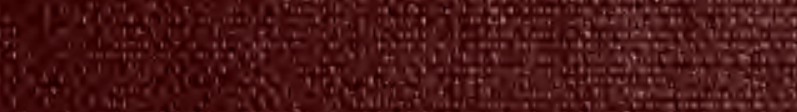

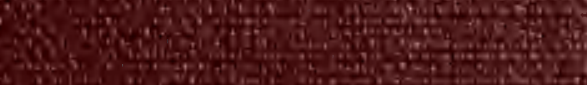

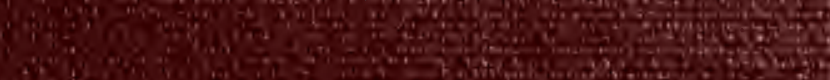

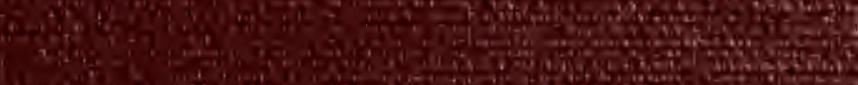
2. 65 (1) (n) Wiv

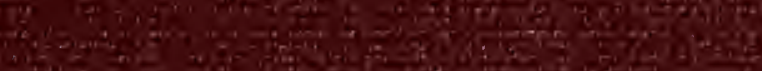
H.

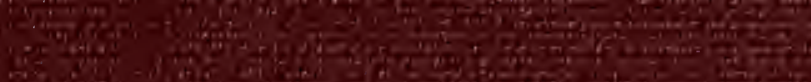

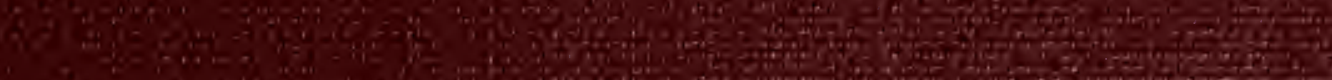

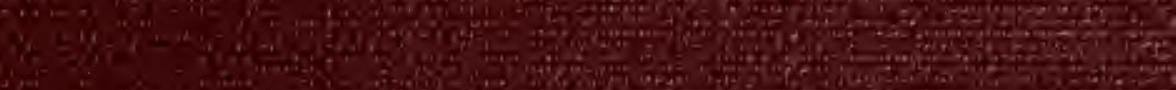

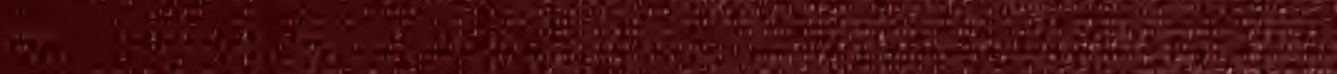
Q (15)

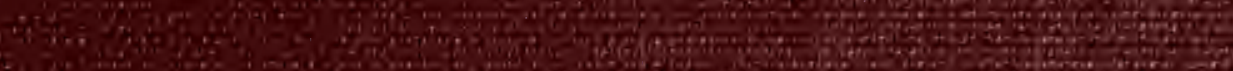
W 6. S6"

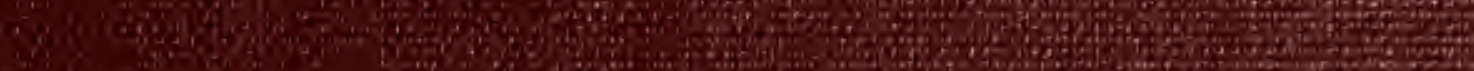
6.

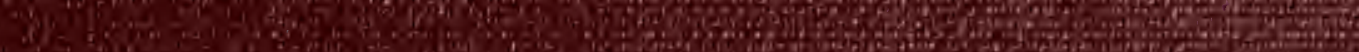

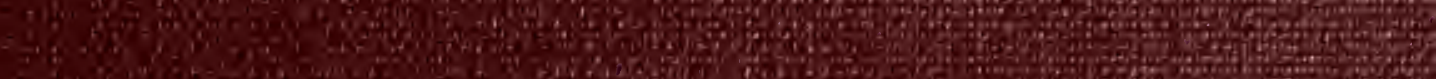
E.

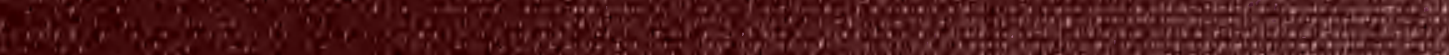


LIBRARY

"UYA ONTARIO MUSEITX 
Digitized by the Internet Archive in 2011 with funding from Royal Ontario Museum 




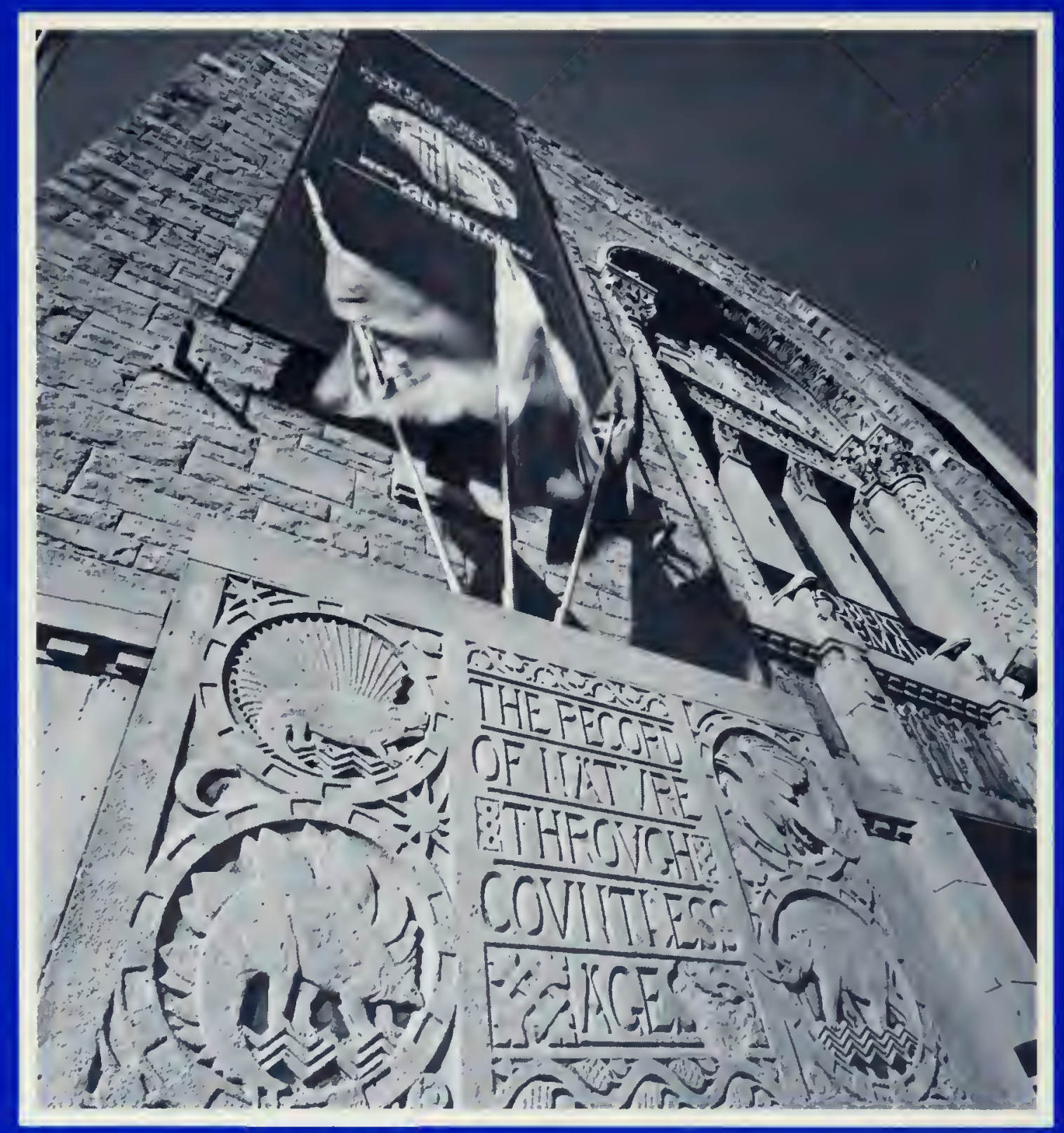



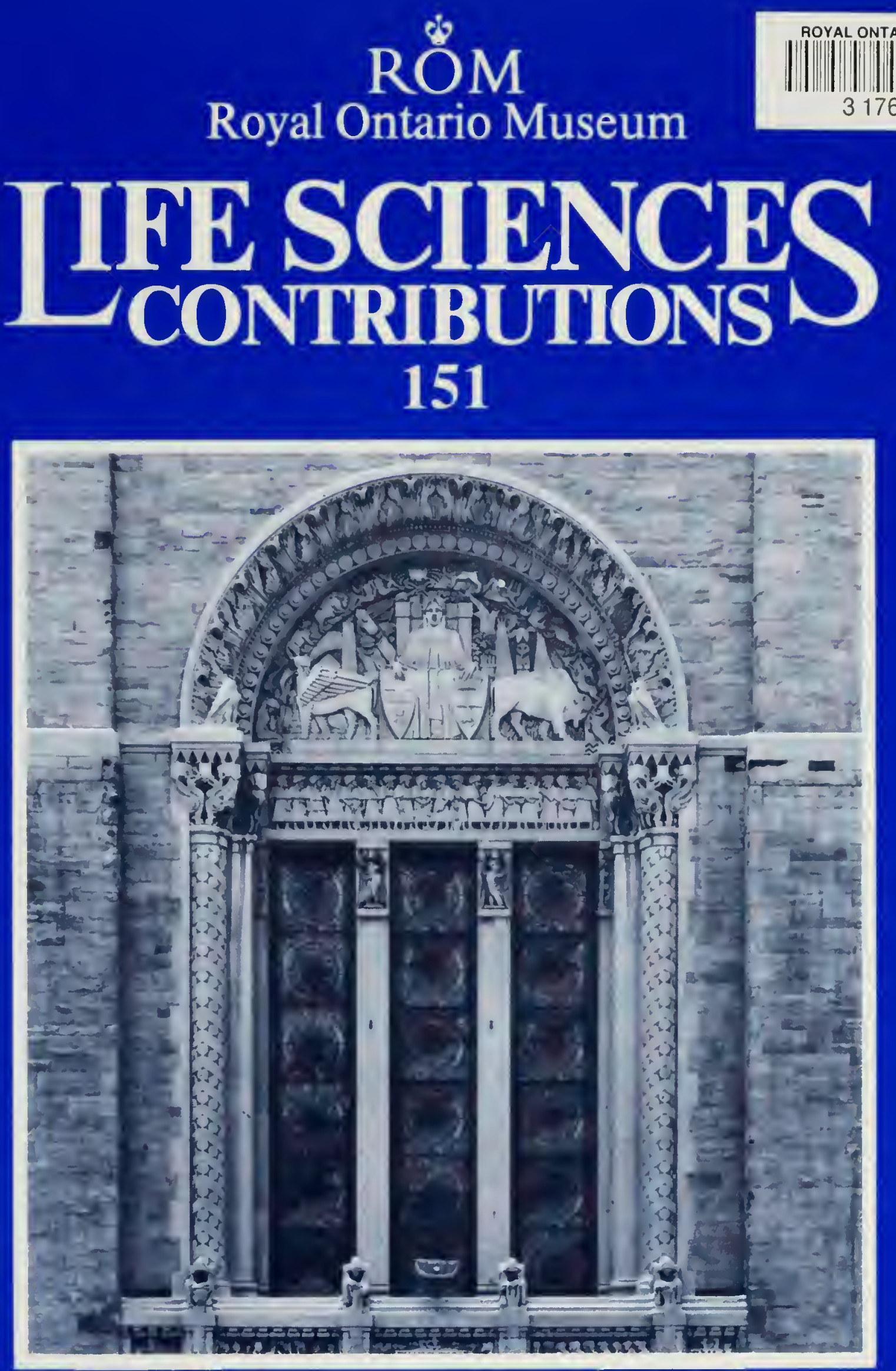

The Structure of the Call Note System of the Warbling Vireo

\author{
Daryl Howes-Jones \\ and \\ Jon C. Barlow
}




\section{ROYAL ONTARIO MUSEUM LIFE SCIENCES PUBLICATIONS INSTRUCTIONS TO AUTHORS}

Authors should prepare their manuscripts carefully according to the following instructions; failure to do so will result in the manuscript's being returned to the author for revision. All manuscripts are considered on the understanding that they are not currently offered for publication elsewhere.

1. General Papers for publication are accepted from ROM staff members and research associates, and from researchers reporting on work done with ROM collections. Monographs on the flora and/or fauna of Ontario may be considered for publication by authors not affiliated with the ROM. Financial contributions towards publication will be welcome. Authors are expected to write clearly and concisely and to omit any material not essential for an understanding of the main theme of the paper.

2. Format Manuscripts (including captions, synonymies, literature cited, and tables) should be typed with double space on 11 " $\times 8 \frac{1}{2}$ " paper with a $1 \frac{1 / 2}{2}$ " margin on all sides. Three xerox copies should be submitted to the Senior Editor of the Editorial Board; the original should be retained by the author(s). The submission should include a separate sheet giving the author(s) names and affiliations, the title of the publication, the series for which it is submitted, the number of typed pages, the number of tables, and the number of plates or figures. Manuscripts should normally be organized in the following order: Contents, Abstract, Introduction, Materials and Methods, Results, Discussion, Conclusions, Summary (if manuscript is long), Acknowledgements, Appendices, and Literature Cited. Authors are encouraged to include foreign-language translations of the Summary, if appropriate. Main headings should be centred; subheadings should be left-justified to the text margin. The first line of the first paragraph in each new section should not be indented. Literature citations in the text should be in the form "Jones (1972)" or "(Jones, 1972)" or "(Smith, 1960:71-79, fig. 17)".

3. Standard Sources The primary authority on questions of format and style is Guide to Authors, available from ROM Publication Services. For matters not covered in the Guide, consult CBE Style Manual (Fifth Edition). Other standard sources are as follows: for
English spelling, The Concise Oxford Dictionary; for Canadian place names and coordinates, Canada Gazetteer Atlas; for the spelling of geographic names, The Times Atlas.

4. Abstract All papers must be preceded by a short, factual abstract, about one per cent of the text in length. The abstract may be followed by four to six key words in parentheses.

5. Taxonomy The name of a taxon should be given in full in headings, at the beginnings of paragraphs, and at its first occurrence in the text. Give the authority and date, if appropriate, with the first mention of each taxon, but not thereafter. Taxonomic papers, particularly synonymies, should follow the layout in Life Sciences Contributions beginning with No. 136. International Codes of Biological Nomenclature must be followed.

6. Literature Cited A complete list of references, in alphabetical order of authors, must be given at the end of the paper. When two or more works of one author are cited, they should be listed chronologically. The names of journals should not be abbreviated. For correct bibliographic form, see Life Sciences Contributions beginning with No. 136 .

7. Tables All tables should be typed on separate sheets and numbered consecutively in arabic numerals in the order of their first mention in the text. Mark the location of each table in the margin of the text.

8. Plates, Figures, and Text-figures Illustrations may be designated according to the conventions of the author's discipline; in some disciplines grouped photographs of scientific subject matter are commonly termed Plates, while line drawings and locality and other illustrations that occupy a full page or less are Text-figures. Usage must be consistent throughout the paper. A full-page illustration for a Contribution, with its caption, should be sized to fit an area of $17.3 \times$ $22.75 \mathrm{~cm}$; for Occasional Papers, the area is $14.1 \times$ $21.2 \mathrm{~cm}$. If captions are lengthy, they may be placed on the facing page. A scale or magnification factor should be included. Authors are reminded that when illustrations are reduced magnification factors will change, and that they are responsible for the conversion. For details, see Guide to Authors. 


\title{
The Structure of the Call Note System of the Warbling Vireo
}

\author{
Daryl Howes-Jones \\ and \\ Jon C. Barlow
}


The Royal Ontario Museum publishes three series in the Life Sciences. CONTRIBUTIONS: a numbered series of original scientific publications.

OCCASIONAL PAPERS: a numbered series of original scientific publications, primarily short and of taxonomic significance.

MISCELLANEOUS PUBLICATIONS: an unnumbered series on a variety of subjects.

All manuscripts considered for publication are subject to the scrutiny and editorial policies of the Life Sciences Editorial Board, and to independent refereeing by two or more persons, other than Museum staff, who are authorities in the particular field involved.

\section{LIFE SCIENCES EDITORIAL BOARD}

Senior editor: D.C. Darling

Editor: R.W. Murphy

Editor: J.H. McAndrews

External editor: C.S. Churcher

Manuscript editor: E.J. Crossman

Production editor: J.E. Hawken

Daryl Howes-Jones is a doctoral graduate of the Department of Zoology, University of Toronto, and this paper formed part of his original dissertation. He currently resides at 152 Don Valley Drive, Kitchener, Ontario, N2P IB2.

Jon C. Barlow is curator, Department of Ornithology, Royal Ontario Museum, and professor, Department of Zoology, University of Toronto.

\footnotetext{
Canadian Cataloguing in Publication Data

Howes-Jones, Daryl, 1951-

The structure of the call note system of the warbling vireo

(Life sciences contributions, ISSN 0384-8159; 151)

Bibliography: $p$.

ISBN 0-88854-343-3

1. Warbling vireo. 2. Bird-song. 3. Vireos - Behavior.

I. Barlow, Jon C., I935- . J1. Royal Ontario Muscum.

III. Title. IV. Series.
}

QL696.P2945H69 $1988 \quad 598.8^{\prime} 71 \quad$ C88-094340-8.

Publication date: 15 December 1988

ISBN 0-88854-343-3

ISSN 0384-8159

(C) Royal Ontario Muscum, 1988

100 Queen's Park. Toronto, Canada M5S 2C6

PRINTED AND BOUND IN CANADA AT THIE ALGER PRESS 


\section{Contents}

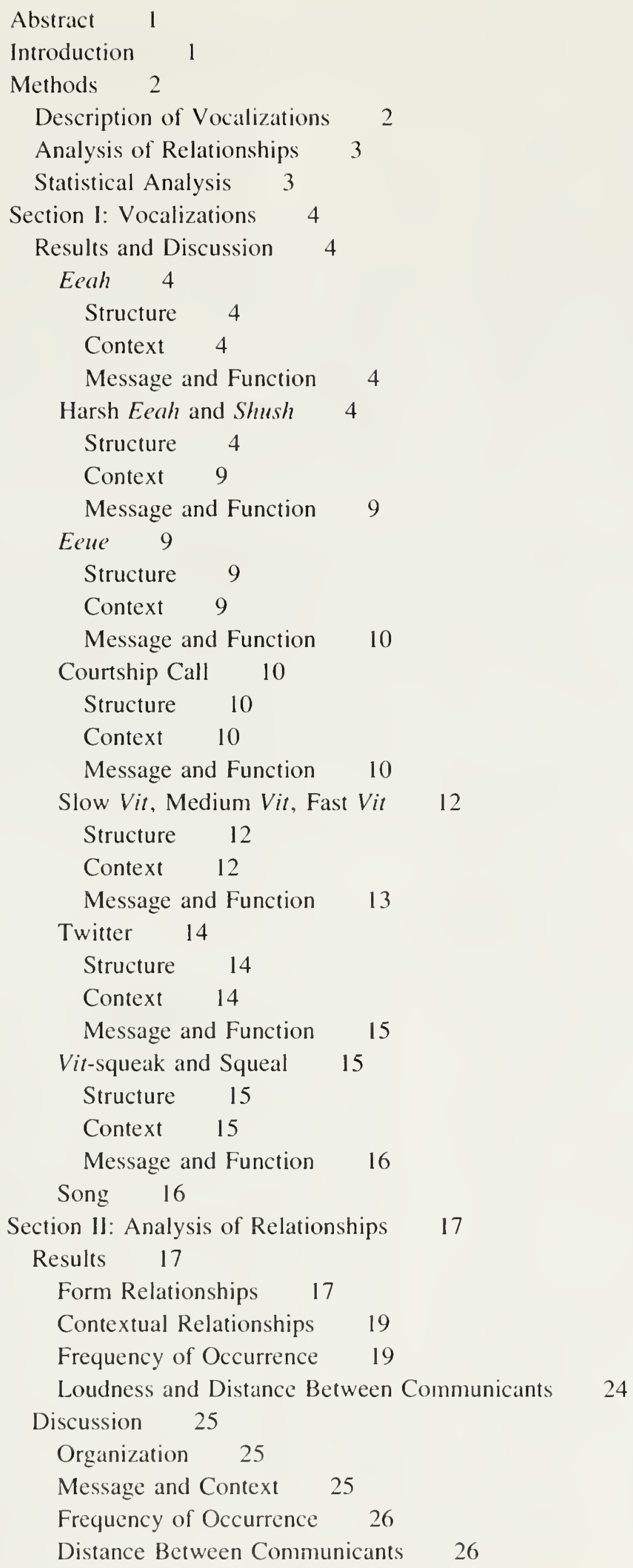


Form and Motivation 27

Structure and Interpretation

Structure and Observer Interpretation

28

Self-Organizing and Interactive Character of the Call Note System

Evolution of the Call Note System

29

Summary and Conclusions

30

Acknowledgements $\quad 30$

Appendices

31

Literature Cited

33 


\title{
The Structure of the Call Note System of the Warbling Vireo
}

\begin{abstract}
This study presents a detailed description of the structure of the call note system of the Warbling Vireo, Vireo gilvus gilvus, in southern Ontario. Properties or attributes used to describe calls are form, context, message, frequency of occurrence, and function. Relationships among calls with respect to each of the attributes and among the attributes are described. Attribute relationships are interdependent. and understanding of form and context relationships is facilitated by use of cluster and principal component analyses.

The call system is graded in form and is organized into two call complexes and five distinct transition series. Each transition series is associated with a different behavioural tendency. Two classes of calls are identified: general-social-vocalizations and criticalsocial-vocalizations. In the former group, calls are more frequent in occurrence, have less precise messages, are more dependent on context for their interpretation, have a wider range of functions, and are less loud with respect to distances between interactants than in the latter group.

A number of constraints influence the structure of the call system including limited processing capacity, distance between communicants, motivational-structural rules, form, and interpretation. The design constraints as well as the interdependencies shown among call attributes are general and can be applied to descriptions of other avian call repertoires. In this study, a set of rules is generated that may govern the organization of vocal repertoires. The call note system is considered to be interactive and self-organizing. General-social-vocalizations are considered more primitive in origin and more resilient to change than critical-social-vocalizations.

Keywords: Vireo gilvus, call note system, form context, message, frequency of occurrence, function, multivariate analyses, general message, critical message.
\end{abstract}

\section{Introduction}

Vocal repertoires are commonly described as systems. Although this term generally implies the existence of components, relationships, and structure (Harary and Batell, 1981), system characteristics have not been described in detail for any avian vocal repertoire. The usual approach is to describe vocal repertoires as aggregates of signals. The description and organization of bird song has been well studied (Isaac and Marler, 1963; Lemon. 1968, 197I; Hinde. 1969: Thompson, 1972; Armstrong, 1973; Kroodsma. 1977; Smith et al. 1978; Thorpe and Hall-Craggs, 1976; Kroodsma and Miller, 1982). In contrast, there have been fewer attempts to elucidate relationships among, or the organization of, calls (but see Marler, 1956; Konishi. 1963: Andrew. 1964: Jenni et al., 1974: Smith, 1966. 1977; Huxley and Wilkinson, 1977). Detailed descriptions of organization exist only for some calls (e.g., Andrew, 1961a) or for selected properties of calls (Marler, 1955. 1967; Smith. 1977: Morton. 1977, 1982).

In this study, a system is defined as a set of objects that possess certain attributes (properties of objects) and includes the relationships that exist among the objects and among their attributes (Hall and Fagen, 1975). The notion of structure is inseparable from any definition of system. A system has structure if compositional rules, laws, or principles exist that impose order on the variety of objects over and above the collection of individual properties (Piaget, 1970: Krippendorf. 1975).

The notion of system and structure is central to the examination of any living entity. The approach has been well developed in the literature of general systems theory (Bertalanffy, 1950, 1968, 1975: Koestler and Smythies, 1969: Laszlo. 1972, 1975; Piaget, 1970, 1971: Sutherland, 1973; Miller, 1978; Cavallo, 1979). Complex systems are extremely difficult to describe in detail. They can be studied using a variety of alternative approaches (for revicws see Piaget. 1970, 1971: Laszlo, 1972: Sutherland. 1973: Krippendorf, 1975: Rapoport, 1975: Miller, 1978; Cavallo, 1979). The tremendous plasticity and flexibility shown 
in behavioural systems preclude mechanistic explanations (Tinbergen, 1951; Baerends, 1976). In this study a "soft" systems approach is adopted (Krippendorf, 1975; Rapoport, 1975) because it is uselul for describing natural language systems (Krippendorl, 1975). A soft system must have structure and consist of many constituent elements that have some property in common (Krippendorf, 1975). The properties or attributes of the call notes used in this study include form, context, message, frequency of occurrence, and function. Apart from the identification of the components (i.e., vocal categories), the relationships among calls and among the attributes of calls (i.e., between context and message) can be described. A soft system also must be characterized by rules of composition or patterns among vocal categories and among attributes.

This study describes the calls and structure of the call note system of the Warbling Vireo (Vireo gilvus) in a population in southern Ontario. The Warbling Vireo is a territorial, insectivorous, migratory, and monomorphic passerine inhabiting parkland and open forest throughout much of North America within forested and parkland regions (Godfrey, 1986; see Barlow, 1980).

This study aims (1) to describe the form, context, frequency of occurrence, probable messages, and functions of calls; (2) to describe the relationships among vocalizations with respect to each of these attributes and to describe relationships among attributes; (3) to determine the structure of the vocal communication system and to identify the design principles responsible for that structure. The song of $V$. gilvus has been described elsewhere (HowesJones, 1985c). Calls and song are compared only to facilitate understanding of the former.

\section{Methods}

Warbling Vireos were studied by Howes-Jones in Cootes Paradise Sanctuary, Royal Botanical Gardens, Hamilton, Wentworth Co., Ontario, from May through August in 1975, 1976, and 1977. Observations were made each day between 06:00 and 20:00. Four pairs of vireos in 1975, seven in 1976, and four in 1977 were studied for I to 7.5 $\mathrm{h}$ each day. Supplementary observations lasting from 5 to 20 min were made on another eight pairs in 1975, and nine pairs in 1976. In total, approximately $1450 \mathrm{~h}$ were spent in the field.

Behaviour and vocalizations were scribed in coded form on data sheets marked out in minutes and seconds. During each observation period, an attempt was made to record all calls given by Warbling Vireos. For each vocalization, the following were recorded: (1) the relationship of the interactants (e.g., members of a pair or neighbouring males); (2) the estimated distance between interactants and between them and the nest; (3) the general context; (4) any concurrent displays; (5) whether the caller was perched or in flight; and (6) if the caller was perched, whether this was immediately before or immediately after flight. If a calling Warbling Vireo was both distant from and not exchanging vocalizations with other conspecifics, then the calling bird was categorized as "alone."

In 1976 and 1977 an assistant also made field observations. When nestbuilding began, one observer remained at the nest and recorded behaviour, while notes on birds away from the nest were made by the other worker. The assistant usually followed a male and recorded song activity. Males sang almost continuously throughout most of the nesting cycle; however, some ceased to sing during the nestling period (see Howes-Jones, 1985a). Except in the latter situation, sex determinations could be made cas- ily during the nesting cycle. An intraspecific encounter was defined as an interaction between a Warbling Vireo and a non-mate conspecific, involving one or more of the following: chase, supplant, or an exchange of vocal and visual signals in close proximity. An interspecific encounter was designated when a Warbling Vireo responded to an intruder by supplanting attack, by chasing, or by uttering mobbing calls (i.e., harsh eeahs).

Observations were made with $7 \times 35 \mathrm{~mm}$ binoculars and a $\times 25$ spotting scope. Vocalizations were recorded with a Gibson 46-cm-diameter parabola and microphone and a Uher $4000-\mathrm{L}$ tape recorder at $19 \mathrm{~cm} / \mathrm{sec}$. A portable cassette tape recorder was also used to record field notes. Tapes of vocalizations were analysed with a Kay-Elemetric Co. 6061-B Sona-Graph at the wide-band setting.

\section{DESCRIPTION OF VOCALIZATIONS}

Unless otherwise indicated, characters and terms used to describe and compare relationships among vocalizations follow Marler (1969) and Shiovitz (1976). "Duration" refers to the length in seconds of figures in each call. In determining the lower and upper frequency limits and bandwidths, only the darkest portions of a figure were measured. In some cases the harmonics of vocalizations are not easily separated and are referred to as "energy bands." "Delivery" is used to denote the number of figures per call, the number of calls per bout, and the patterning of bouts and figures over time. The term "sound form" includes the sound of a vocalization as it was heard, the shape of figures, and the similarity in shape among individual birds. 
ANALYSIS OF RELATIONSHIPS

An attempt was made to describe and quantify the contexts in which calls were uttered. Three categories were distinguished. The first category, referred to as immediate-ongoing-activity, included the following: whether the call was given when the bird was perched, whether the call was given just before or just after flight and while the bird was perched, or whether the bird called in flight and what the distance of flight was. The number of times a call was given in each of the above contexts was tabulated and expressed as a per cent. When the contexts were not quantified, an estimate was made from observations compiled in the field. Estimates were denoted by ' $\mathrm{X}$ ' if the vocalization occurred $30 \%$ of the time or more, by ' $x$ ' if the call occurred less than $30 \%$ of the time, and by '- ' if it occurred less than $5 \%$ of the time.

The second category, referred to as immediate-environmental-situation, described the proximity of interactants to each other and of the calling bird to the nest in question when a call was given. When the distances for each call were tabulated and arranged in frequency distributions (not shown). they were found to resemble negative exponential curves. The medians of the frequency distributions were calculated excluding the values when the bird was alone. Also computed were the percentage of calls uttered within $5 \mathrm{~m}$ of another bird, the range of distances between interactants (excluding the highest $5 \%$ to eliminate extreme values), and the percentage of calls uttered while a bird was alone.

The third category of context, referred to as generalenvironmental-situation, included aspects of the general setting and the relationships among interactants. Types of interactions included interspecific nest defence, non-mate conspecific interactions, and interactions between mates.

Smith $(1969,1977)$ suggested that most vertebrates encode messages from a finite set of message classes. The messages of the calls of the Warbling Vireo were categorized using Smith's (1977:87-126) classification.

An estimate of the frequency of occurrence of calls was made from data for six pairs in 1976 and four pairs in 1977. If calls were given in rapid succession or in bout form (i.e., see vits), then each bout was assumed to encode the message and was noted as a single signal.

The "carrying distance" of a vocalization was defined as the distance between a bird and the observer at which attenuation made the call inaudible. Estimates of carrying distance were determined under favourable environmental conditions and in territories where acoustic interference from other birds was negligible. In selected territories, distances between prominent trees or between known perch sites were paced off or measured with a measuring tape. Carrying distances were estimated based on comparisons with these distances.

"Referent distance" of a vocalization was defined as the median of the distribution of distances between communicants when a vocalization was heard. It is an estimate of the distance between communicants when a signal was given. The median was used as the measure of central tendency because distributions resembled negative exponential curves.

\section{STATISTICAL ANALYSIS}

Statistical comparisons of similar characters in different calls were made using the $t$-test and approximate $t$-test (Sokal and Rohlf, 1969). The latter test was used when sample variances were heterogeneous, as determined by the F-max test. A Wilcoxon two-sample test (Sokal and Rohlf, 1969) was used to test for differences between the means of two samples that were not normally distributed. Chi-square tests (Sokal and Rohlf, 1969) were used to test for independence in two-way contingency tables. Probability ranges were designated as follows: $* * *=\mathrm{P} \leqslant$ $0.001, * *=\mathrm{P} \leqslant 0.01, *=\mathrm{P} \leqslant 0.05$.

Multivariate analysis was used to compare form and context relationships among vocalizations using 17 and 28 multistate and meristic characters, respectively. Characters were standardized and correlation coefficients for all pairs of vocalizations computed. From this matrix of correlations, the first three principal components were extracted and all vocalizations were projected and plotted onto these three components. Character loadings were computed for each of the first three components to identify the common sources of variation in the data. Cluster analyses using the unweighted pair-group method with arithmetic averages (UPGMA) were performed on distance matrices for both sets of data; the results were summarized in phenograms. Cophenetic correlation coefficients were calculated to assess degree of concordance between the similarity values among calls implied by the phenogram and those calculated from the original data.

The multivariate analyses were performed using the NTSYS package of programs developed by Rohlf, Kishpaugh, and Kirk (for details of methods see Sneath and Sokal, 1973). Multivariate analysis was carried out on an IBM 370 computer at the University of Toronto Computing Services. 


\section{Section I: Vocalizations}

RESULTS AND DISCUSSION

Eight calls and male song were recognized in the first field scason. In subsequent years it was possible to discern differences between calls using context, message, lunction, and frequency of occurrence. These attributes were used to distinguish 12 calls.

\section{Eeah}

\section{STRUCTURE}

Eeah (Table I, Fig. IA,B) was a frequently uttered call, with a nasal quality, given by both sexes. Eeahs consist of a series of harmonics of variable, but generally broad, frequency range. Harmonics of most eeahs are not parallel or equidistant from each other, indicating production by two acoustic sources (Greenewalt. 1968). Amplitude is greater in the second half of the call. Eeahs average 0.5 sec in duration.

Variation in energy-band structure and pitch occurred among individual vireos. Although the eeahs of some birds showed similar patterns on different days, suggesting that individual differences exist, sample sizes were too small to test for this.

\section{CONTEXT}

Eeahs were given in a variety of contexts which were divisible into two classes: (1) interactions with the mate or young, and (2) interactions with non-mate conspecifics and other species. In male-female interactions, several contexts were distinguished. Prior to pairing, males sang and gave eeahs regularly and at least once in eight of nine observations periods (i.e., periods during which birds were constantly observed) during which they patrolled their newly established territories. Eeahs were exchanged in 65 of 105 observation periods during the establishment of pair bonds. During the pre-nestbuilding period, birds often gave eeahs prior to or after a flight when mates were nearby. When mates were separated, one bird, usually the male. called loudly and continuously. Other calls uttered at this time included the twitter, rapid vit, and vit-squeak (see later sections). Calling continued until the mate appeared or responded vocally. During this period, $44 \%(\mathrm{~N}=240)$ of eeahs occurred when mates were separated and apparently searching for each other

During the incubation period, both sexes gave eeahs without apparent stimuli. Such spontaneity suggested that birds were maintaining contact with mates. 1ncubating females also uttered this call in response to male song. Occasionally. mates engaged in loosely synchronized cxchanges: female eeahs and "courtship calls" with male song (courtship call).

From the start of incubation, eeahs were given by mates exchanging places on the eggs. Birds gave this call on
$26.2 \%(\mathrm{~N}=1915)$ of their trips to the nest and $12.5 \%$ $(N=1918)$ lrom the nest. Late in the nestling period when the young were about to lledge, fenales uttered ceahs a few metres from the nest.

Although the large majority of eeahs was given while a bird was perched $(98.3 \%, \mathrm{~N}=230)$, most of these occurred just before or after flight. In 1975, 75.8\% ( $\mathrm{N}=$ 380) of ecahs recorded during the nestbuilding period occurred immediately before or after flight. In 1977, 24.6\% $(\mathrm{N}=180)$ of eeahs recorded prior to nestbuilding occurred inmediately before or after flight. Of 41 eeahs sampled in the pre-nestbuilding period. $63.4 \%$ were uttered immediately before or after a flight of more than $10 \mathrm{~m}$.

\section{MESSAGE ANI FUNCTION}

Prior to pairing, eeahs appeared important for establishing contact between potential mates. Continued use after pairing and throughout most of the nesting cycle may help to maintain and reinforce the pair bond. Eeahs given with long-distance flight may allow mates to monitor each other's position and direction of movement. Eeahs may act as a greeting when birds approach one another. During nesting. eeahs coupled with vits function to coordinate exchanges of birds on and off the nest. Late in the nestling period. eeahs uttered near the nest may have stimulated the young to leave the nest. In low-intensity interactions involving defence of the nest or territory, eeahs likely indicated mild aggression. In potentially aggressive interactions, eeahs appeared to function as a distance-increasing signal. their effect being one of mutual repulsion that restores spacing between individuals (Marler, 1968). In many pair social interactions, eeahs appear to function as a distance-reducing signal, their effect being to bring individuals in closer proximity (Marler, 1968).

Eeah has an imprecise message (Table 2). It provides information that may identify a caller, possesses structural properties (vertical spectrograms, sudden onset) that may help a recipient locate the caller. and identifies probabilities for interaction. Thus, the eeah may be termed a general, medium- to long-distance social and locative call.

The eeah is very similar in form to the mial call of the Red-eyed Vireo ( $V$. olivaceus) and the ehhh of the Philadelphia Vireo (V. philadelphicus) (Lawrence. 1953: Barlow and Rice. 1977). These calls are both given in contexts similar to those of the eeah and also have locative and aggressive functions.

\section{Harsh Eeah and Shush \\ STRUCTURE.}

The harsh eech (Table 1. Fig. IC) grades in form with the eeah and is distinguished by its greater loudness, higher 


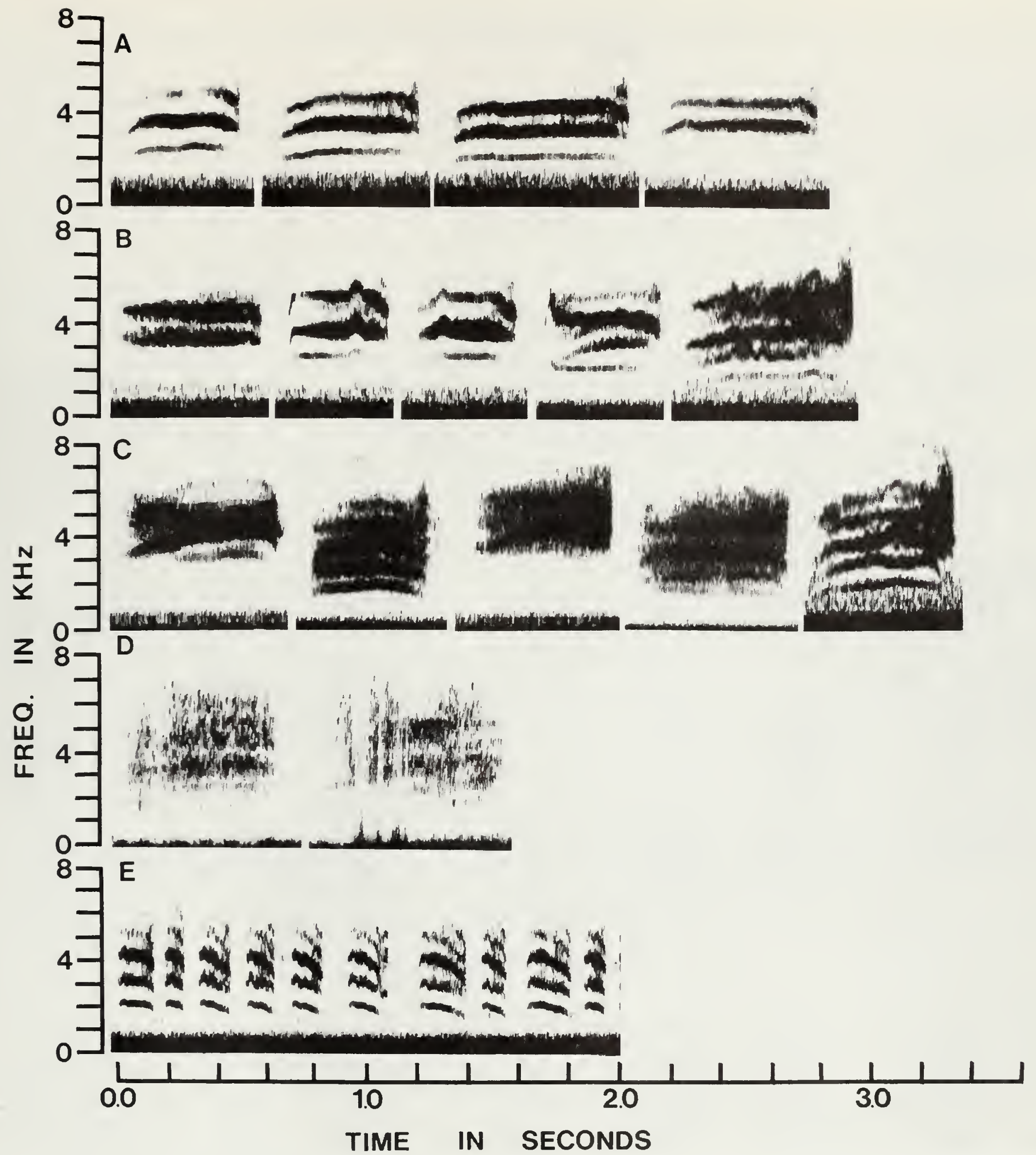

FIG 1. Warbling Vireo calls: $A$, eeahs given by the same individual over a 5 -min period: $B$, eeahs given by different individuals; $C$, harsh eeahs; $D$, shush: $E$, a portion of a bout of eeue. 
T'A131. 1. Characteristics of calls of the Warbling Vireo.

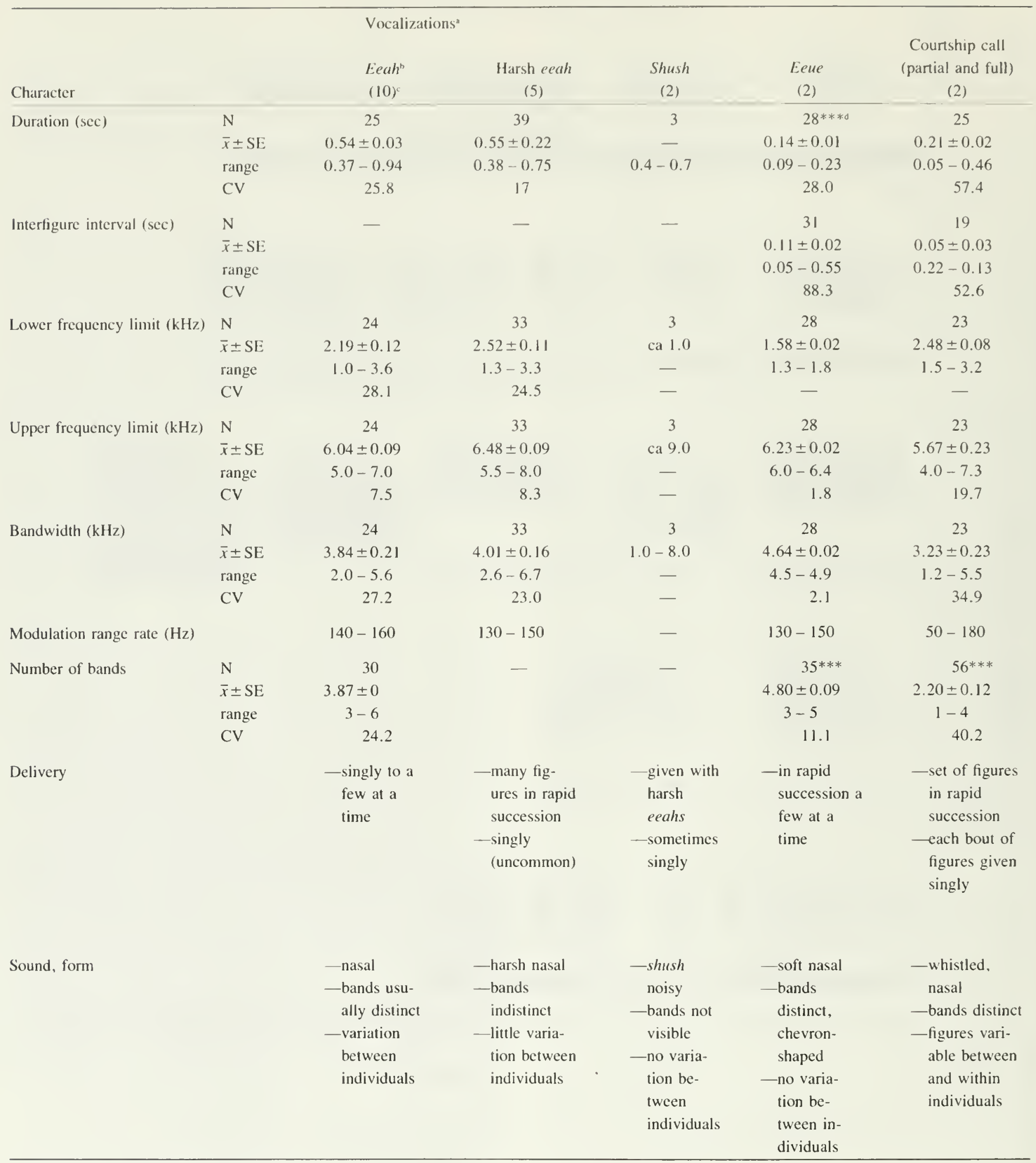

Tests used were $t$-test and approx. $t$-test.

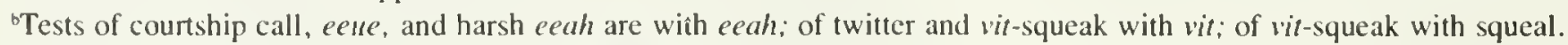

'Individual sample sizes are indicated in parentheses.

$\stackrel{\mathrm{s}}{\mathrm{P}} \leqslant 0.05=* ; \mathrm{P} \leqslant 0.01=* * ; \mathrm{P} \leqslant 0.001=* * *$. 


\begin{tabular}{|c|c|c|c|c|c|}
\hline Slow vit & $\begin{array}{l}\text { Medium vit } \\
\text { (6. pooled results) }\end{array}$ & Fast vit & Twitter ${ }^{b}$ & $\begin{array}{c}\text { Vit-squeak } \\
\text { (3) }\end{array}$ & Squeal ${ }^{b}$ \\
\hline 47 & same & same & $56^{* * *}$ & $38 * * *$ & $54 * * *$ \\
\hline $0.026 \pm 0.001$ & & & $0.037 \pm 0.001$ & $0.055 \pm 0.005$ & $0.125 \pm 0.008$ \\
\hline $0.015-0.038$ & & & $0.031 \pm 0.054$ & $0.01-0.19$ & $0.04-0.28$ \\
\hline 27.6 & & & 16.6 & 60.9 & 46.4 \\
\hline- & - & - & 52 & 32 & $43 * *$ \\
\hline - & - & - & $0.028 \pm 0.002$ & $0.235 \pm 0.025$ & $0.139 \pm 0.013$ \\
\hline $0.3-1.0$ & $0.1-0.23$ & $0.06-0.09$ & $0.01-0.067$ & $0.03-0.69$ & $0.05-0.36$ \\
\hline - & - & - & 42.5 & 60.6 & 46.4 \\
\hline 49 & same & same & $43 * *$ & $36^{* * *}$ & $48 *$ \\
\hline $1.7 \pm 0.09$ & & & $2.19 \pm 0.07$ & $2.26 \pm 0.09$ & $2.73 \pm 0.12$ \\
\hline $0.05-2.7$ & & & $1.5-3.2$ & $1.4-3.9$ & $1.3-4.8$ \\
\hline 37.1 & & & 10.9 & 25.3 & 31.3 \\
\hline 49 & same & same & 43 & 36 & 48 \\
\hline $7.19 \pm 0.12$ & & & $7.38 \pm 0.12$ & $6.77 \pm 0.19$ & $6.87 \pm 0.16$ \\
\hline $4.9-8.5$ & & & $5.2-8.5$ & $5.0-9.0$ & $4.2-9.0$ \\
\hline 22.7 & & & 19.7 & 16.8 & 16.2 \\
\hline 49 & same & same & 43 & $36^{* * *}$ & 48 \\
\hline $5.48 \pm 0.18$ & & & $5.23 \pm 0.13$ & $4.47 \pm 0.21$ & $4.15 \pm 0.20$ \\
\hline $2.8-8.0$ & & & $2.6-6.7$ & $2.6-6.8$ & $1.7-6.1$ \\
\hline 22.7 & & & 15.9 & 29.1 & 33.4 \\
\hline- & - & - & - & - & - \\
\hline 30 & same & same & 49 & 47 & $60 * * *$ \\
\hline $3.93 \pm 0.21$ & & & $4.16 \pm 0.21$ & $3.47 \pm 0.21$ & $2.33 \pm 0.18$ \\
\hline $2-6$ & & & $2-7$ & $1-7$ & $1-6$ \\
\hline- & & & - & 42.4 & 60.8 \\
\hline $\begin{array}{c}\text { - } 1 \text { to } 4 \text { per sec } \\
\text {-bouts given a } \\
\text { few at a time } \\
\text { to continuous } \\
\text { — single bouts } \\
\text { uncommon }\end{array}$ & $\begin{array}{l}\text {-5 to } 8 \text { per sec } \\
\text {-bouts given a } \\
\text { few at a time } \\
\text { to continuous } \\
\text { - single bouts } \\
\text { uncommon }\end{array}$ & $\begin{array}{l}\text {-9 to } 13 \text { per } \\
\text { sec } \\
\text {--bouts given } \\
\text { a few at a } \\
\text { time to } \\
\text { continuous } \\
\text { - single bouts } \\
\text { uncommon }\end{array}$ & $\begin{array}{l}\text {-15 per sec } \\
\text { - figures given } \\
\text { in bouts } \\
\text { - each bout } 0.8 \\
\text { to } 3.0 \mathrm{sec} \\
\text { duration }\end{array}$ & $\begin{array}{l}\text {-rate same as } \\
\text { for slow and } \\
\text { medium vits }\end{array}$ & $\begin{array}{l}\text { - a few at a time } \\
\text { to continuous }\end{array}$ \\
\hline $\begin{array}{l}\text { —metallic } \\
\text { —chevron-shaped } \\
\text { or slurred } \\
\text { upwards } \\
\text {-bands usually } \\
\text { distinct } \\
\text { - no variation } \\
\text { between } \\
\text { individuals }\end{array}$ & —same & —same & $\begin{array}{l}\text { - metallic, } \\
\text { twitter } \\
\text {-chevron- } \\
\text { shaped } \\
\text {-no variation } \\
\text { between } \\
\text { individuals }\end{array}$ & $\begin{array}{l}\text { —a few at a } \\
\text { time } \\
\text { —metallic to } \\
\text { squeal } \\
\text { - figures vari- } \\
\text { able between } \\
\text { and within } \\
\text { individuals }\end{array}$ & $\begin{array}{l}\text { —squeal, high- } \\
\text { pitched } \\
\text { —variable } \\
\text { between } \\
\text { and within } \\
\text { individuals }\end{array}$ \\
\hline
\end{tabular}




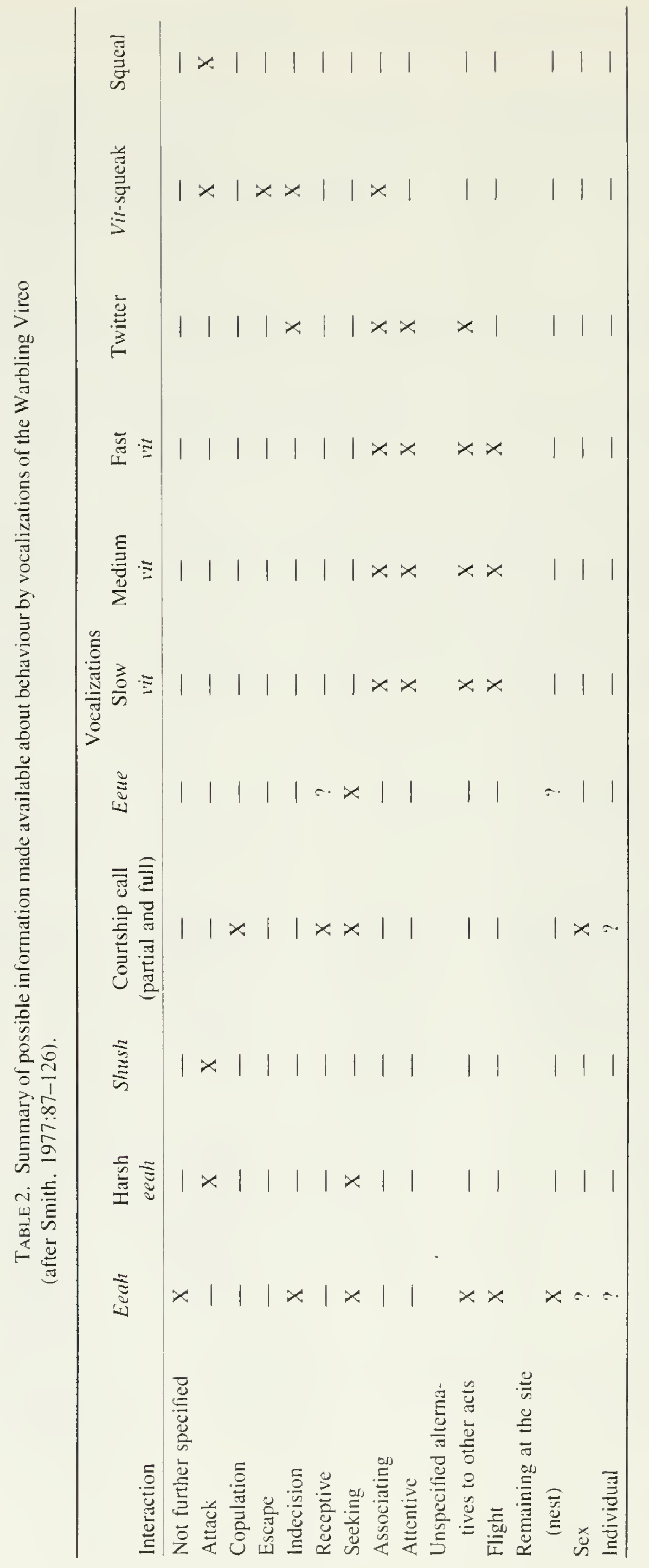


pitch, and harsher sound. Whereas harsh ceahs were given repeatedly (100 per $\mathrm{min})$, eeahs were given singly or a few at a time.

Harsh eeahs appear as a band of energy between 1.3 and $8.0 \mathrm{kHz}$. When the harmonic structure could be distinguished, sound production appeared to be by two acoustic sources. Both the mean lower and the mean upper frequency limits of the harsh eeah were significantly higher $\left(t=1.983^{*} ; t=3.296^{* *}\right.$, df $=55$, respectively; see Table 1) than those of the eeah. Comparisons made on half-speed recordings of harsh eeahs and eeahs showed the former to have a slightly lower modulation rate. When mates gave harsh eeahs together, individuals were often distinguishable by differences in their calls.

Shush calls (Table 1, Fig. 1D) grade in form with harsh eeahs. Intense harsh eeahs may have a shush-like quality. Spectrographically, the shush resembles white noise. Most energy is between 1.0 and $8.0 \mathrm{kHz}$.

\section{CONTEXT}

Harsh eeahs were given when a potential predator approached the nest. For example, Blue Jays (Cyanocitta (ristata) and Common Grackles (Quiscalus quiscula) elicited a strong response. A hundred or more harsh eeahs could be given if an intruder remained near or continued to approach the nest. Small passerines evoked a weaker response (i.e., fewer calls). Generally, when an intruder was first supplanted or had harsh eeahs directed towards it, it flew away. If a number of harsh eeahs were given in succession, conspecifics (the mate or neighbours) and sometimes other species flew to the site of the encounter.

Eeahs and harsh eeahs were given at least once in $75 \%$ $(\mathrm{N}=237)$ of interspecific encounters and in $75 \%(\mathrm{~N}=$ 61 ) of aggressive intraspecific encounters involving males. Harsh eeahs usually occurred in proximity to the intruder and immediately before or after a movement to attack. A significantly greater percentage of harsh eeahs $(99.3 \%, \mathrm{~N}$ $=295)$ than eeahs $(23.5 \%, \mathrm{~N}=1171)$ occurred within $5 \mathrm{~m}$ of another interactant $\left(\chi^{2}=571.031 * * *, \mathrm{df}=1\right)$. A few harsh eeahs $(8.5 \%, \mathrm{~N}=95)$ were given at or just prior to contact with an intruder.

The shush was given singly and usually only at the time of physical contact with an intruder $(95 \%, \mathrm{~N}=49)$. A significantly larger percentage of shushes (100\%) were uttered within $1 \mathrm{~m}$ of another interactant than were harsh eeahs $\left(31.2 \%, \mathrm{~N}=295, \chi^{2}=82.264^{* * *}\right.$. $\left.\mathrm{df}=1\right)$. Shushes not given at contact occurred during intense mobbing.

Harsh eeahs were uttered during aggressive interactions between males. When the intensity of an interaction increased, eeahs increased in number and loudness and graded into harsh eeahs. The persistence, proximity, size, and species of intruder near the nest appeared to determine the frequency and occurrence of the eeah.
Loud eeahs and harsh eeahs were given by birds apparently searching for their mates (see Eeah. p. 4). This was the only situation not involving aggression where harsh eeahs were given.

\section{MESSAGE AND FUNCTION}

Harsh eeah and shush convey a more intense and specific message than eeahs (Table 2). The first two calls indicate a high probability of attack and function as high-intensity scolding or mobbing calls. The loudness and repetitiveness of calls appear to be the cues that attract conspecifics and other species to the site of encounter to mob the intruder. As a corollary, harsh eeahs may facilitate contact between widely separated mates when one bird is searching for the other. In the latter situation, harsh eeahs may encode a message to approach.

\section{Eeue}

\section{STRUCTURE}

The eene (Table 1, Fig. 1E) grades in form with the eeah. Eenes sounded like a short, soft eeahs. The "full" form of this utterance consisted of many calls given in rapid succession. This form was heard 3 times. The interval between calls ranged from 0.05 to $0.55 \sec (\mathrm{N}=31)$. The "partial" eeue was heard 19 times and was intermediate in sound between the sounds of a full eeve and an eeah. The partial eeve occurred in bouts of two to five calls. Both sexes gave partial eeues (males 10 times, females 7 times, sex unknown 6 times). Only males were heard giving the full eeue.

Eeues were less variable in form than eeahs. Eene calls consist of five distinct harmonics. The harmonics are chevron-shaped and descend in pitch. Two acoustic sources are apparent. The modulation rate $(130$ to $150 \mathrm{~Hz}$ ) was similar to that of the harsh eeah. The lower frequency limit and bandwidth of the eeue call were both significantly less variable $\left(\mathrm{F}_{\max }=22.611^{* * *}\right.$ and $109.635^{* * *}$, respectively $)$ and larger (approx. $t=4.9385^{* * *}$ and $3.793 * * *$, respectively). The duration of the eeue $(0.14 \mathrm{sec}, \mathrm{N}=28)$ was both significantly less variable $\left(\mathrm{F}_{\max }=6.259 * * *\right)$ and shorter (approx. $t=14.171 * * *$ ) than that of the eeah call.

\section{CONTEXT}

The contexts of 22 eeue calls were as follows: (1) prior to nestbuilding, 9 calls ( 5 by males, 1 by female. 3 by unknown sex) were recorded when mates foraged within $4 \mathrm{~m}$ of each other: (2) from nestbuilding onwards, 13 eeuc calls were recorded when a bird was within $2 \mathrm{~m}$ of the nest-site or on the nest. The 13 eeue calls occurred as follows: 4 ( 2 by females, I by male, 1 by unknown sex) during courtship activities; 2 uttered by males at an abandoned nest and 1 by a male while a female was selecting a nest-site; the remaining 6 ( 4 by lemales, 2 by males, 1 
by unknown sex) near the nest-site during nestbuilding or at the start of incubation.

When full eeues were given, birds crouched low on a branch and fluffed their breast and flank feathers. Once, a male gave eelues for 15 min interspersed with vit-squeaks and distorted song (see later sections). Birds giving eelle calls did not show approach-avoidance behaviour, nor did mates show any apparent response. It was difficult to determine the factors important in eliciting the eeue. Proximity of the mate and nest, particularly during the late prenestbuilding and nestbuilding periods, appeared to be important, but other factors evoking this call have not been identified.

\section{MESSAGE AND FUNCTION}

The message of the eeue was difficult to categorize (Table 2). Birds giving eeue calls appeared nonaggressive and submissive. The eeue was given when a caller was near its mate or its nest at the time of nestbuilding, suggesting that the call encodes either information concerned with unspecified alternatives to nest-oriented activities or association with the nest (see Smith, 1977). Because the call was associated with the nest and courtship, it may function as a precopulatory solicitation signal.

\section{Courtship Call}

STRUCTURE

Courtship calls (Table 1, Fig. 2) grade in form with eeahs, but in a manner distinct from either the harsh eeah or the eeue. Calls most closely resembling eeahs in form were termed "partial" courtship calls (Fig. 2A). They comprise one or more distorted eeah-like calls and a series of shorter figures that resemble portions of eeahs separated by intervals of about $0.05 \mathrm{sec}$. Calls of more than five figures are termed "full" courtship calls (Fig. 2B). The longest courtship call was about $2.5 \mathrm{sec}$. Courtship calls have a fractured, quavering, nasal sound that is higher in pitch than the eeah. Only females give courtship calls.

Courtship call figures were significantly shorter $(t=$ $9.189^{* * *}$ ) in duration than individual eeahs and were variable in form. Frequency modulation tended to decrease in shorter figures. Courtship call figures have fewer harmonics (one to three) than eeahs. As with the eeah, harmonics from two acoustic sources were sometimes evident.

Some variants of the courtship call had figures with pure tones or few energy bands (Fig. 2C). These calls were heard by us from a few females at the nest during the incubation and nestling periods and when the males were nearby and singing. These calls were frequently heard during the loosely synchronized duets involving courtship calls and male song.

Fig. 2C-E illustrates exchanges of male song and female courtship calls recorded over a 5-min period. Initially, the male was about $5 \mathrm{~m}$ from the nest. After every few songs, the female responded with an eeah less than $0.5 \mathrm{sec}$ after the end of the last song of the group. The female also gave distorted eeahs and partial courtship calls (Fig. 2C) interrupting a male either before his song was finished or immediately after he had sung (Fig. 2D,E). When these latter two calls were recorded, the members of the pair were within $1.5 \mathrm{~m}$ of each other. Most figures of courtship calls differed from those in the male's song repertoire.

\section{CONTEXT}

Courtship calls were given most often in the nestbuilding period. Male presence, song activity, and proximity to the nest appeared to affect the female's propensity to utter courtship calls. Most courtship calls $(60.4 \%, \mathrm{~N}=53)$ were given when the male was within $10 \mathrm{~m}$ of the female, and in all circumstances the male was singing. Almost all courtship calls $(74 \%, \mathrm{~N}=50$ ) were given within $10 \mathrm{~m}$ of the nest.

Copulatory behaviour and full courtship calling peaked on the fourth day of nestbuilding (3.24 attempted copulations per hour, 2.82 courtship calls per hour. $N=12$ h). Full courtship calls were usually given by females when a singing male was nearby, just prior to or after a copulatory attempt. In contrast, partial courtship calls characterized episodes of less intense courtship activity, or when the female was more than $10 \mathrm{~m}$ from the nest. The partial courtship call was not always associated with a copulatory behaviour.

Prior to nestbuilding, partial courtship calls were given during intense inter-pair interactions and encounters with conspecific interlopers, but seldom during interactions between mates. During the incubation and nestling periods. partial courtship calls were occasionally heard when the female was on or near the nest and the male began singing nearby.

\section{MESSAGE AND FUNCTION}

Courtship calls identified the caller as female, and indicated a desire to interact and a willingness to engage in copulatory activity (Table 2). The message of the partial courtship call was less specific than that of the full courtship call. The association between courtship calls and copulatory activity suggests that the calls function as a precopulatory solicition signal. The partial call appeared to indicate a lower level of sexual arousal than the full call. In encounters between neighbouring pairs, partial calls may identify a bird as female and thereby prevent attacks by males. The synchronized exchanges of courtship calls and song during the incubation period may help maintain the pair-bond and facilitate exchange of partners at the nest. 


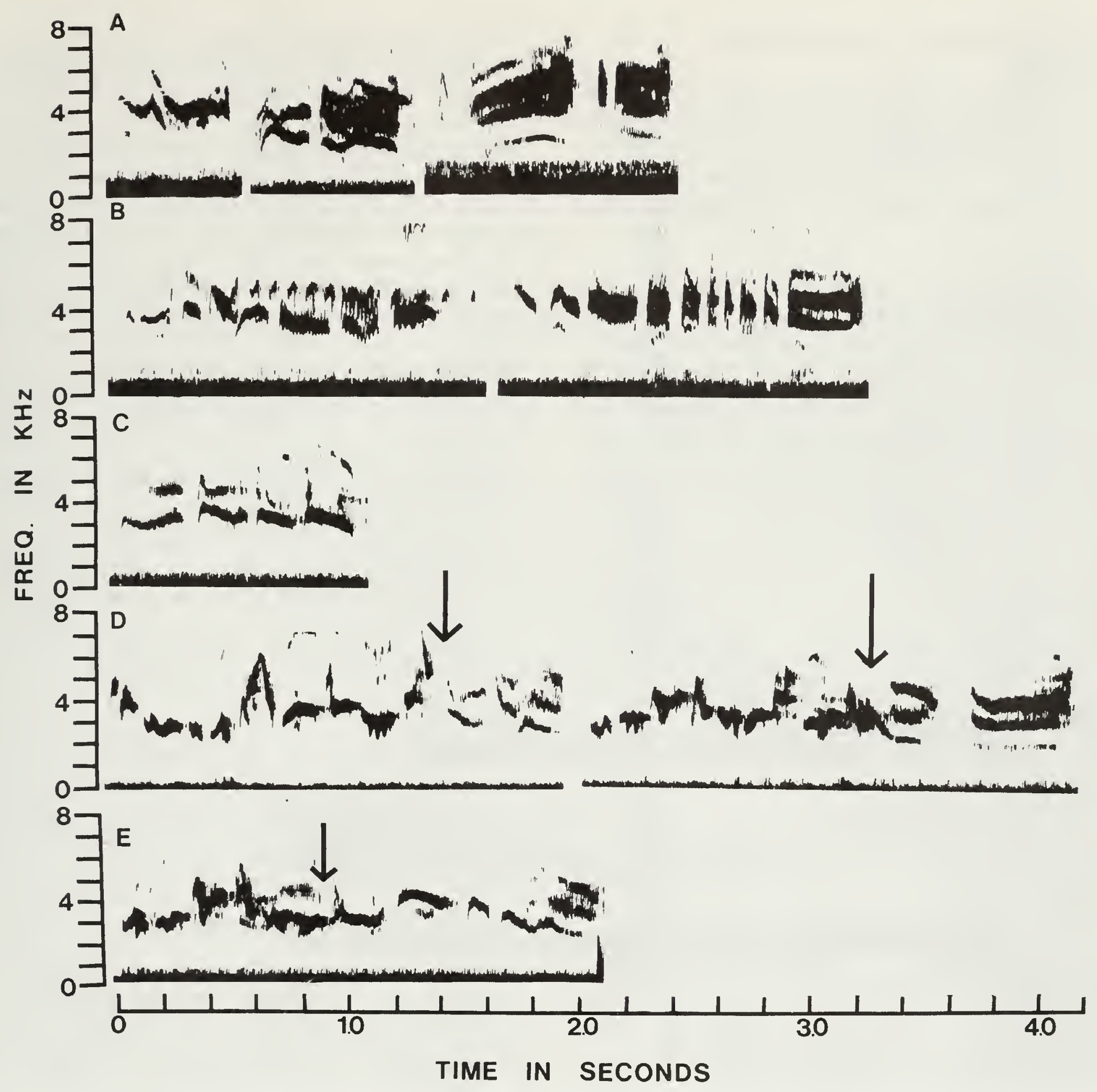

Fig. 2. Female courtships calls: $A$, partial courtship call; $B$, full courtship call; $C$, whistled courtship call; $D, E$, figures to left of arrows are of male song, figures to right of arrows are of partial courtship calls. 


\section{Slow Vit, Medium Vit, and Fast Vit} STRUCTURE

Slow, medium, and fast vits (Table I, Fig. 3A-C) form a vocal continuum and are distinguished by their rate of delivery. Each consists of a series of vit notes averaging $0.026 \mathrm{sec}(\mathrm{N}=47)$ in duration. A vit consists of two to six chevron-shaped energy bands. Vit calls have a metallic sound.

Slow vits comprise 1 to 3 figures per second; medium vits, 4 to 8 figures per second; and fast vits, 9 to 13 figures per second. A few fast vits may well have exceeded 13 figures per second; however, such calls were rare and not taped. When the vit rate exceeded 13 notes per second, it graded into a twitter (see next section). Slow vits were usually uttered in short bouts of only a few figures. If a bird uttered vits continuously, they were more likely to occur at a medium to fast rate. Bouts of medium and fast vits usually lasted several seconds. During intense social interactions, birds gave vits throughout the encounter.

\section{CONTEXT}

Most vits ( $80 \%, \mathrm{~N}=526$, all forms) were uttered in flight, some began prior to flight, and some continued after landing. Slow vits (Fig. 4) usually characterized short flights of a few metres in length $(2.1 \pm 0.4 \mathrm{~m}, \mathrm{~N}=231)$. Slow vits often occurred when mates were in close proximity $(3.0 \pm 0.3 \mathrm{~m}, \mathrm{~N}=192)$. Slow vits were also given when mated Warbling Vireos were foraging together, or flying to and from the nest. During pair formation and the prenestbuilding period, slow vits were exchanged between mates almost continuously. During nesting, the females gave slow vits on the nest in apparent response to male song. When a lone bird gave slow vits $(13.5 \%, \mathrm{~N}=222)$, it appeared to be searching for its mate or was near the nest.

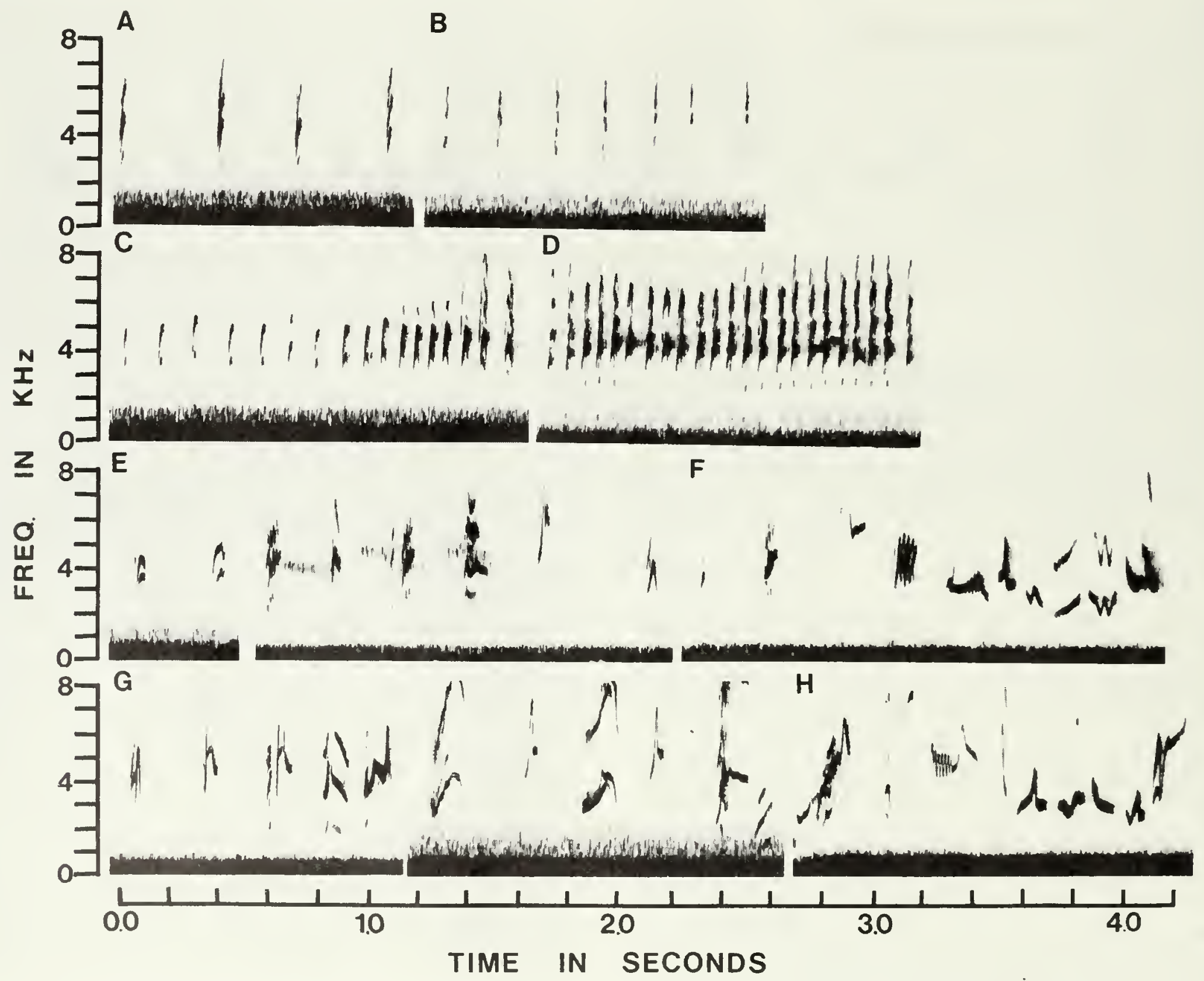

FlG. 3. Warbling Vireo calls: $A$, slow vit; $B$, mediun vit: $C$, fast vit grading into a twitter: $D$, twitter: $E$. vit-squeaks: $F$, the first four figures are vit-squeaks oceurring with figures of male song: $G$, squeals: $H$. the first four figures are squeals occurring with figures of male song. 
The mean distance of flight during which medium vits were given was $18.3 \pm 1.9 \mathrm{~m}(\mathrm{~N}=269$ : Fig. 4). Vits were uttered in $16.9 \%(\mathrm{~N}=2068)$ of trips to the nest and in $35.6 \%(\mathrm{~N}=2054)$ of trips away from the nest. Of trips where vits were uttered, medium vits were given on most $(61.0 \%, \mathrm{~N}=1457)$, with slow and fast vits given on $36.2 \%$ and $2.7 \%$, respectively. Medium vits were associated with all male-male territorial interactions witnessed. Medium vits were given by lone birds seeking interaction with their mates $(10.6 \%, \mathrm{~N}=274)$, by the female at the nest and engaged in courtship behaviour, or by the pair near nestlings.

Of the three calls, fast vits were least associated with flight (Fig. 4). Significantly more fast vits than medium lits were given $\left(\chi^{2}=14.376^{* * *}\right.$, $\left.\mathrm{df}=1\right)$ while the bird was perched. Fast vits occurred during copulation, during male-male territorial interactions involving physical contact, and during pair-pair territorial interactions. Lone birds that gave fast vits $(10 \%, \mathrm{~N}=50)$ were members of either sex seeking their mates or were females involved in courtship near the nest. The mean distance between communicants $(1.3 \pm 0.4 \mathrm{~m}, \mathrm{~N}=53)$ when a fast vit was given was significantly less than the mean distance between birds uttering medium vit $(6.8 \pm 0.7 \mathrm{~m}, \mathrm{~N}=245)$ or slow vit (3.1 $\pm 0.3 \mathrm{~m}, \mathrm{~N}=192$ ) calls (smallest $\mathrm{U}=8418.5^{*} * *$, Wilcoxon test)

In summary, vits occurred in a broad range of contexts. Although vits were given during most flights, there were exceptions. Unmated and lone males rarely gave vits in flight. Birds rarely uttered vits in flight when attacking an animal near the nest. Warbling Vireos stealing nest material from the nests of other species or conspecifics, or males intruding into a neighbour's territory, did not call in flight. In contrast. the probability that a bird would vit was high when a bird was in its own territory close to its mate or its nest. In general, the duration and rate increased when interacting conspecifics were unfamiliar with each other (i.e.. initial encounter between potential mates or interactions with neighbours) or with the intensity of the encounter (i.e.. territorial or courtship encounters as opposed to foraging together). The relative proportions of each type of vit with respect to their frequency of occurrence. flight distance, and context are summarized in Fig. 5.

\section{MESS AGE AND FUNCTION}

Delivery rates of vit may provide information concerning the location of the caller, the probability of flight, and the length of a flight (Table 2). Slow and medium vits appeared to maintain the cohesion of a pair during foraging and allow a pair to coordinate other activities. Thus vits may be related to the tendency to associate or remain with another bird (see Smith. 1977). As such, they function as a proximity-maintenance signal between individuals (Mar-

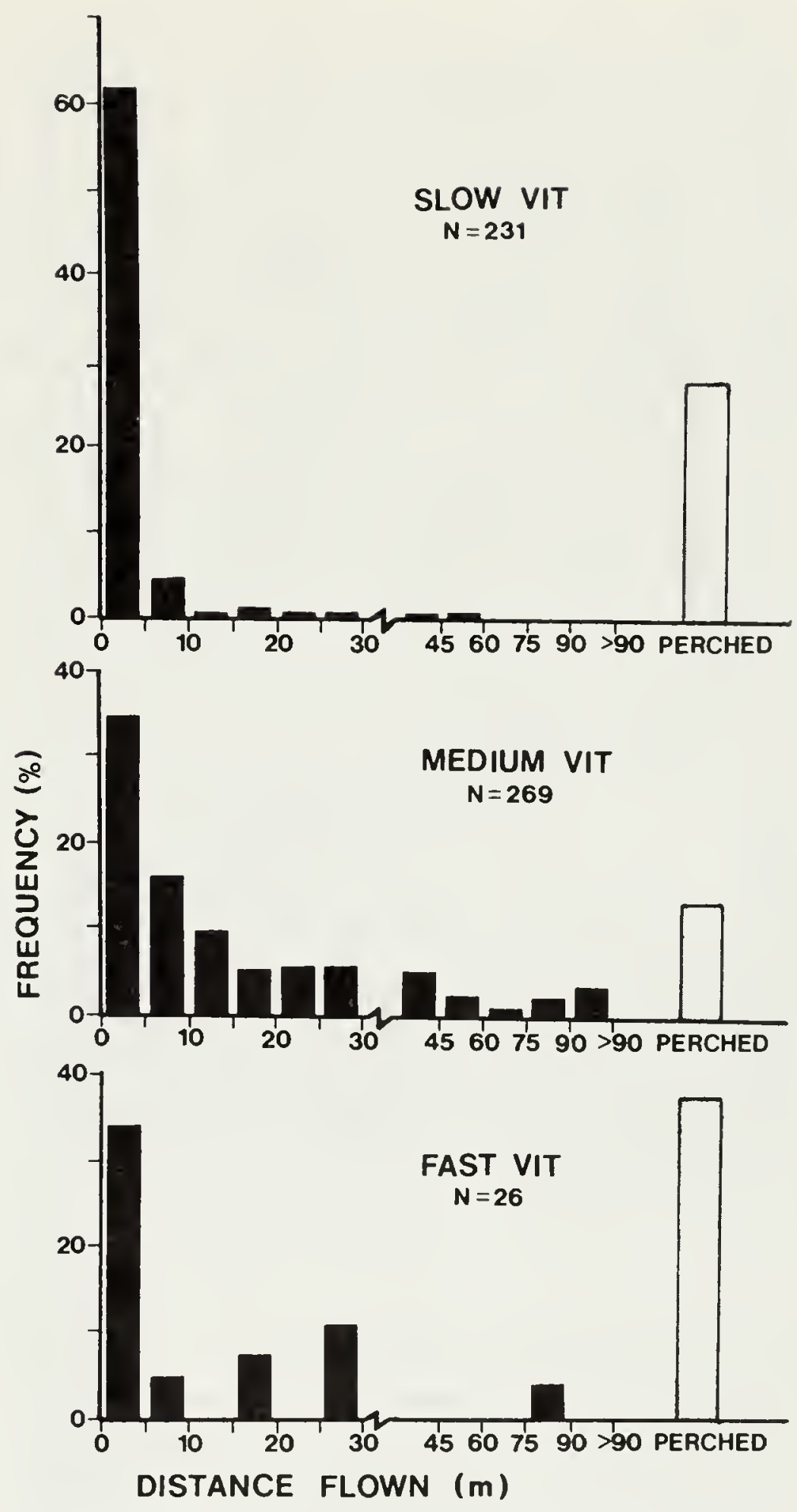

Fig. 4. The frequency of occurrence of vits and its relationship to the distance flown (in metres) by the calling bird when vit was given in flight. Open bars indicate situations in which birds gave vits while perched. Samples were taken before nestbuilding. Numbers of observations noted.

ler, 1968). When mates were separated or searching for one another, the louder and more prolonged eeah was used to maintain contact.

Although a vit may not encode a specific behavioural alternative, increased rates of vits may inform the recipient of the probability that a bird will act and approach, or of the degree of excitation of the bird. As such. the giving of vits may be a form of metacommunication (Wilson. 1975:191-193). 


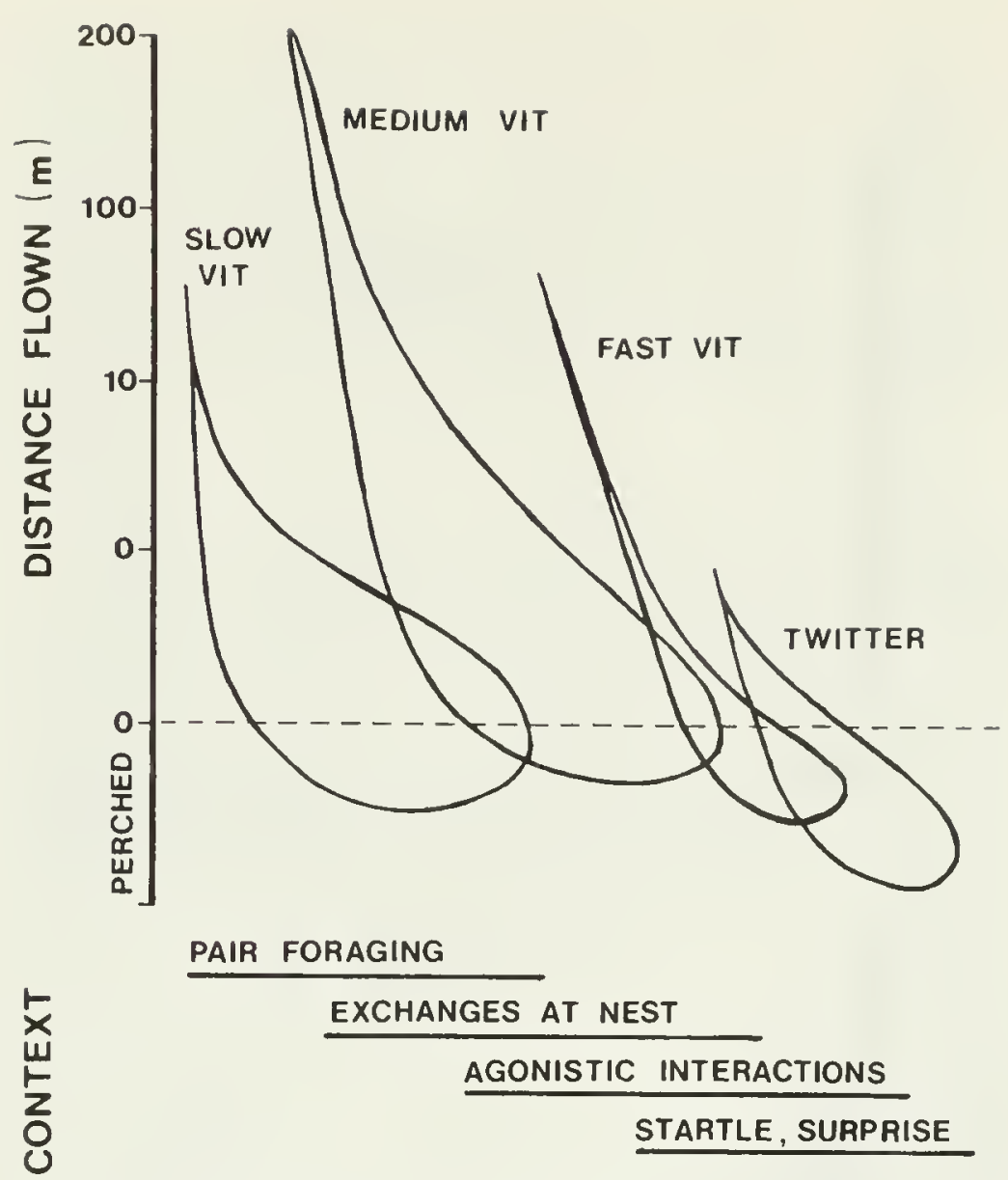

FIG. 5. Schematic representation showing relationships among contexts in which vits and twitters occurred. The area covered by each call is proportional to the frequency of occurrence of the call in that context. Generally, the more highly excited the bird, the greater the rate at which the bird will vit while perched. The line under each context indicates which calls occur in association with that context.

In summary, both slow and medium vits may be termed short-distance locative, contact, or flight calls. The failure of Warbling Vireos to give vits when intruding in a neighbour's territory or during nest defence may be adaptive in that vits might inform the defender or intruder of the proximity and state of flight of other birds. Fast vits likely indicate a high probability of action, with the information on the specific action being conveyed by other contextual sources of information.

The counterpart in Philadelphia and Red-eyed vireos of the vit call in gilvus has been rendered phonetically as "jit" or "it." These calls are low-volume contact calls exchanged between mates when they are together (Bent. 1950; Rice, 1974; Barlow and Rice, 1977).

\section{Twitter}

\section{STRUCTURE}

The twitter (Table 1, Fig. 3D) grades in form with the vit call but can be differentiated from the vit in three ways. First, the basic note of the twitter is significantly longer in duration $(t=9.179 * * *)$ than the vit note. Secondly, the twitter spans a wider frequency range, even though it has a significantly higher lower-frequency limit (approx. $t=4.468 *$ ) than the vit. Within individual notes, more energy is concentrated in the higher harmonics than in vits, giving the twitter its distinctive and higher-pitched sound. Thirdly, the intervals between consecutive notes in the twitter are of equal length, whereas the interval between vit notes is variable. Fast or medium vits may grade directly into twitters (Fig. 2C). Generally twitters were given as discrete calls. Bouts of twitters were estimated to last from 1 to $3 \mathrm{sec}$.

\section{CONTEXT}

Twitters generally occurred in contexts similar to those in which fast and medium vits were heard, with minor differences. Twitters were seldom given in flight or before or after flight. A significantly larger percentage of twitters $\left(81 \%, \mathrm{~N}=121 ; \chi^{2}=19.8601 * * *, \mathrm{df}=1\right)$ than fast vits was given while a bird was perched. Twitters were more frequently associated with alarm or startle situations and intense intraspecific interactions than were vits (see Fig. 5).

Prior to nestbuilding, 70\% ( $\mathrm{N}=100)$ of all twitters occurred when birds were less than $5 \mathrm{~m}$ apart, but after nestbuilding, this percentage dropped significantly $\left(\chi^{2}=\right.$ $17.9802 * * *, \mathrm{df}=1)$ to $37.5 \%(\mathrm{~N}=72)$. This decrease was likely associated with proximity of a caller to the nest. From early nestbuilding to the late nestling period. $60.3 \%$ $(\mathrm{N}=68)$ of twitters occurred within $5 \mathrm{~m}$ of the nest. During the same period, $31 \%(\mathrm{~N}=72)$ of twitters were given by lone birds, and in most cases near the nest. The high percentage of twitters near the nest, during this early nestbuilding to late nestling period, may in part be because of the large amount of time that mates spent within $10 \mathrm{~m}$ of it $(73.8 \%, \mathrm{~N}=1682 \mathrm{~min}$; Howes-Jones, 1985b).

During nestbuilding, twitters occurred when the mates were engaged in precopulatory behaviour near the nest. when the female was alone and approached the nest, or when a female was near the nest and the male appeared or started to sing. Twitters were occasionally heard when a pair was near the nest and exchanging positions to feed or incubate. Adults sometimes twittered at the nest in apparent response to the hatching of the young.

Prior to nestbuilding, $26.9 \%(\mathrm{~N}=78)$ of twitters recorded occurred when a lone bird was searching for its mate. Other instances involved pairing interactions or malemale and inter-pair territorial interactions. Twitters were heard in $59.0 \%(\mathrm{~N}=61)$ of all aggressive intraspecific encounters.

Two components common to all contexts in which twitters occurred were (1) involvement in intraspecific interactions where the bird manifested ambivalence towards attacking or fleeing or engaging in copulatory activity; and (2) a sudden change in the social setting or a startle or 
alarm. Examples include the giving of twitters by females when the male started singing, or when a conspecific approached. The rate of twitter prior to nesting ( 2.97 calls per hour, $\mathrm{N}=24.4 \mathrm{~h}$ ) was almost five times greater than during nesting ( 0.64 calls per hour, $N=208.8 \mathrm{~h}$ ). Perhaps the fact that early in the season mates were least "familiar" with each other and were also subjected to repeated territorial incursions by conspecifics is a partial explanation of the high incidence of twittering at this time.

\section{MESSAGE AND FUNCTION}

The information encoded in the twitter is unclear (Table 2). The transition from slow, medium, and fast vits to twitter appeared to indicate increasing excitement. Vits reflected general associations, while the twitter showed an intense association. After a twitter, birds engaged in a number of behaviours. In potentially aggressive situations, birds near a bird that twittered usually stopped approaching or moved away. A twitter in such situations may warn others to keep their distance (i.e., distance-maintenance signal) and that closer approach may be met with aggression.

\section{Vit-squeak and Squeal}

\section{STRUCTURE}

Figures in vit-squeaks (Table 1, Fig. 3E,F) are variable in shape and resemble distorted vit notes. Compared to the $v$ it, the vit-squeak has a significantly smaller bandwidth ( $t$ $\left.=3.701^{* * *}\right)$, greater lower-frequency limit $(t=4.229 * * *)$, and a longer duration (approx. $t=5.344 * * *$ ). The vitsqueak has a squeaky. sometimes whistlelike sound.

Squeals (Fig. 3G,H) grade in form into vit-squeaks in both sexes or into song figures in males. Squeals and vitsqueaks are distinguishable from each other by rate of utterance. Vit-squeaks were comparable to slow vits and usually heard in combination with slow and medium vits. Whereas each vit-squeak note was aurally distinct, squeals sounded continuous. Squeals tended to be given in longer bouts than vit-squeaks. Squeals sometimes occurred in combination with vit-squeaks or vits; however, most bouts of squealing in males were interspersed with song figures (Fig. $3 \mathrm{H}$ ). In song, squeals appeared as progressive distortions of typical song figures. Vit-squeaks also occurred in song (Fig. 3F). Squeals resembled a high-pitched whistle. Vit-squeaks were distinguished from squeals by their significantly longer duration (approx. $t=7.435^{* * *}$ ) and higher lower-frequency limit (approx. $t=3.060^{*}$ ).

\section{CONTEXT}

Of 65 squeals. 46 were given by males, 4 by females, and 15 by birds of unknown sex. Vit-squeaks were heard in almost all intraspecific encounters when birds were close to each other or were near the nest. In the latter context birds usually showed approach-avoidance behaviour. When compared to all other forms of vitting, a significantly greater (smallest $\chi^{2}=7.344^{*}, \mathrm{df}=1$ ) percentage of vit-squeaks $(68.4 \%, \mathrm{~N}=79)$ occurred while a bird was perched.

Prior to nestbuilding, a significantly higher percentage of vit-squeaks $(100 \%, \mathrm{~N}=53)$ were given within $5 \mathrm{~m}$ of another bird as compared to slow, medium, or fast vits (smallest $\chi^{2}=6.982^{* *}$, $\mathrm{df}=1$ ). Vit-squeaks were occasionally given by unpaired males while patrolling their territories. One male gave vit-squeaks frequently when his mate disappeared (probably lost to a predator) during incubation.

Vit-squeaks occurred frequently at pairing, and much less often after pair formation when aggressive interactions between mates were uncommon. Vit-squeaks were heard when mates were near the nest, during nest exchanges, during nestbuilding, or during courtship. Most vit-squeaks $(97.2 \%, \mathrm{~N}=38$ ) occurred within $5 \mathrm{~m}$ of the nest. Also, most vit-squeaks by females $(89.5 \%, \mathrm{~N}=38)$ were given within $5 \mathrm{~m}$ of the male. Females near the nest appeared to give vit-squeaks in response to male song, or when the young were in the nest.

Squeals were given both in flight and while perched. As birds approached one another or became aggressive, vit-squeaks graded into squeals. Squeals were heard in $41.0 \%(\mathrm{~N}=71)$ of aggressive intraspecific interactions. Table 3 summarizes the contexts of 63 squeals. Fourteen were recorded from mid to late incubation period and involved a lone male away from the nest. No stimuli could be ascertained for the seemingly spontaneous outbursts of squeals combined with song. In summary, the close proximity of unfamiliar conspecifics seemed most important in eliciting these calls.

TaBLE 3. Summary of contexts of 63 instances of squeal.

\begin{tabular}{|c|c|}
\hline $\begin{array}{l}\text { Number of } \\
\text { occurrences }\end{array}$ & Contexts \\
\hline 13 & $\begin{array}{l}\text { - male-male interactions with a chase or } \\
\text { physical contact }\end{array}$ \\
\hline 8 & $\begin{array}{l}\text {-male-male interactions that also involved a } \\
\text { female }\end{array}$ \\
\hline 5 & -two males actively counter-singing \\
\hline 1 & $\begin{array}{l}\text { - male } V . \text { gilvus and male American Red- } \\
\text { start (Setophaga ruticilla) interaction with } \\
\text { a chase and physical contact }\end{array}$ \\
\hline 10 & $\begin{array}{l}\text {-during copulation or active soliciting when } \\
\text { the pair was near or in physical contact }\end{array}$ \\
\hline 6 & $\begin{array}{l}\text { - pair exchanging positions on the nest or } \\
\text { engaged in a chase near the nest }\end{array}$ \\
\hline 6 & $\begin{array}{l}\text { - pair exchanging positions on the nest, } \\
\text { mates not in visual contact }\end{array}$ \\
\hline 14 & - spontancous or unknown reasons (sec text) \\
\hline
\end{tabular}




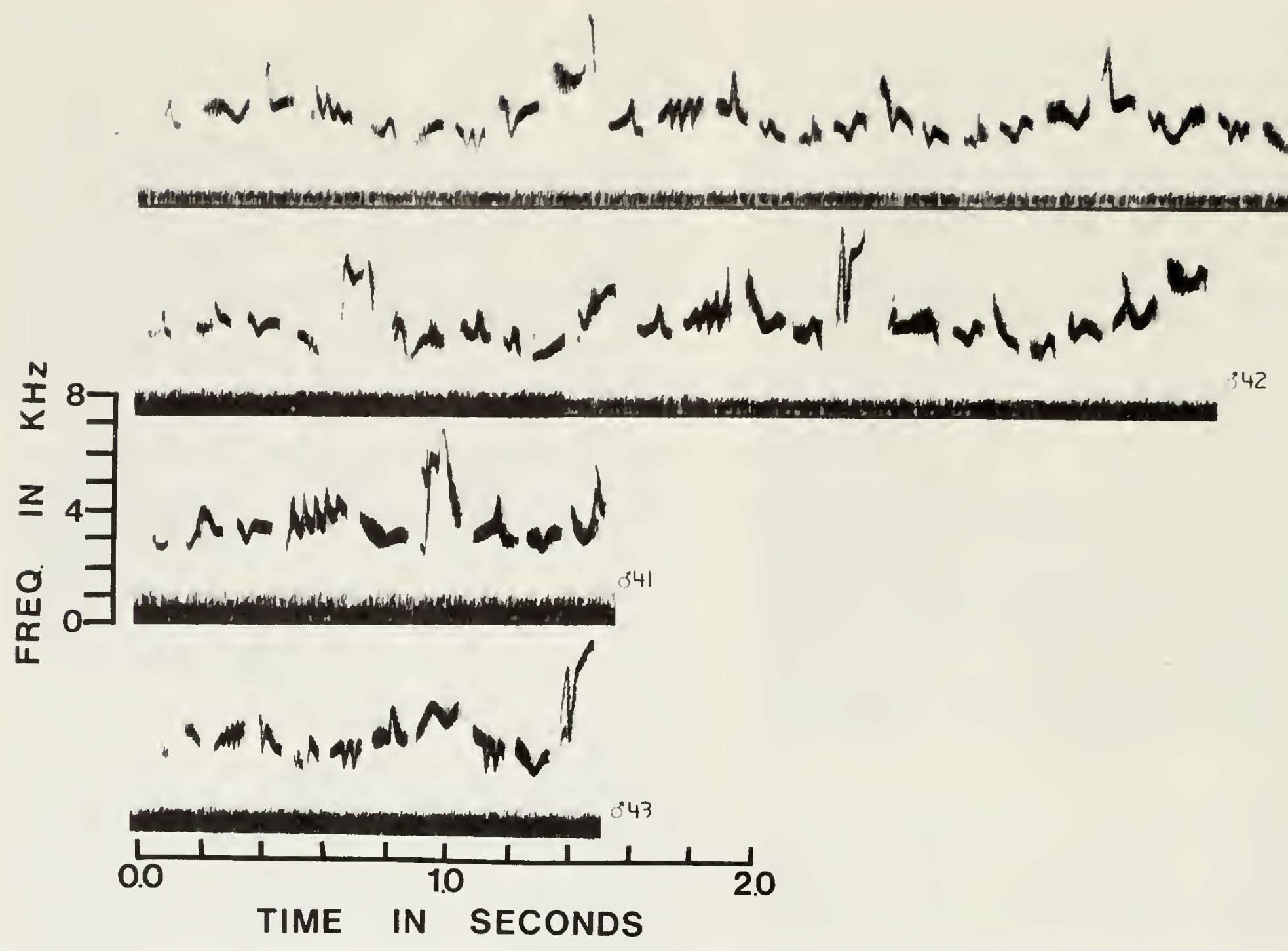

FIG. 6. Examples of song from four male Warbling Vireos.

\section{MESSAGE AND FUNCTION}

The message of the vit-squeak (Table 2) is similar to that of the medium and slow vits; however, the association of the vit-squeak with approach-avoidance behaviour, its reduced association with flight, and its increased association with conspecifics in close proximity indicate a fear component. Squeals appear to represent a more intense state of fear or aggression (Table 2). The call's association with intraspecific aggressive interactions in which birds are in close proximity and in physical contact (i.e., copulation attack) is probable. Vit-squeaks seemingly function as a distance-maintenance signal, and squeals as a distanceincreasing signal. By the late nestling stage, some males almost cease singing. indicating that the aggressive role of song in territorial defense has diminished (Barlow, 1962); during the late nestling stage some males appeared to have difficulty forming song properly and this resulted in distorted or squeal-song.

\section{Song}

The song of the Warbling Vireo (Fig. 6) is complex in structure. It consists of figures of variable shape averaging about $0.12 \mathrm{sec}$ in duration and separated by silent intervals of about $0.04 \mathrm{sec}$. Song duration averaged about $2.4 \mathrm{sec}$ $(N=596)$. Song has a characteristic warbling sound and is distinct from all calls except for squeal song. Squeal song may grade from a minor distortion of song figures in which song is still recognizable into an intense squeal in which song becomes unrecognizable (Fig. 3H). The contextual and structural characteristics of song are described elsewhere (Howes-Jones, 1982, 1985a, 1985c). 


\section{Section II: Analysis of Relationships}

\section{RESULTS}

\section{Form Relationships}

To analyse form relationships among vocalizations, 17 multistate and quantitative variables were identified and subjected to multivariate analysis in order to determine clusterings among vocalizations (Appendices 1,2). A threedimensional principal component analysis (PCA) ordination (Fig. 7) revealed five basic clusters of vocalizations: (1) slow vit, medium vit, fast vit; (2) vit-squeak, squeal; (3) eeah, harsh eeah, shush; (4) twitter, eeve; (5) male song and courtship call. A high proportion of the character variance $(89.9 \%)$ was explained on the first three principal components, and so the three-dimensional plot is a reliable representation of form relationships among vocalizations. Characters with high-factor loadings on PC 1 and PC 2 are general shape, frequency, sound, and delivery. Variables with high values on PC 3 are attributable to interval and sound.

A phenogram based on a matrix of distance coefficients (Fig. 8) gave results similar to those of the PCA. The vit calls, twitter, and eeue were closely grouped. The cophenetic correlation $(0.953)$ was high, indicating that the phenogram accurately depicts the positions of vocalizations in two-dimensional space.

Variants of some vocalizations were not included in the multivariate analysis. Full courtship calls graded in form into partial courtship calls, which in turn graded into eeahs.
Some twitters were found to grade in form from vits. Variants of the eere call showed a transition from the eeah. Based on observation of variant forms and multivariate analysis, a schematic representation was arrayed to show form affinities and the graded character of the call system (Fig. 9). Overall, the calls can be divided into two complexes: an "eeal complex" and a "vit complex." Characters that best separate the two complexes are duration and sound quality. Calls of the eeah complex tend to be longer and have a nasal quality; in contrast, calls of the vit complex are metallic and squeal-like. The eeah and slow and medium vits form the core of each call complex. Other calls radiate from the centres in a graded or "transition series." Each transition series diverges in form as a function of a different set of characters (Table 4). As a result, calls at the ends of each transition series show increasing dissimilarity from those of other transition series in the same complex. Calls that form the converging ends of each complex (i.e., loud eeahs, courtshiplike eeahs, eetre-like eeahs) tend to be similar in form.

Song is regarded as separate from the vit and eeah complexes. Song shows a nested, hierarchical structure organized at a number of levels (Howes-Jones, 1982, 1985c). Two associations appear to exist between song and the call complex. First, song grades with squeal-song, and second, song shows closest similarity to the female courtship call (see Figs. 8, 9).

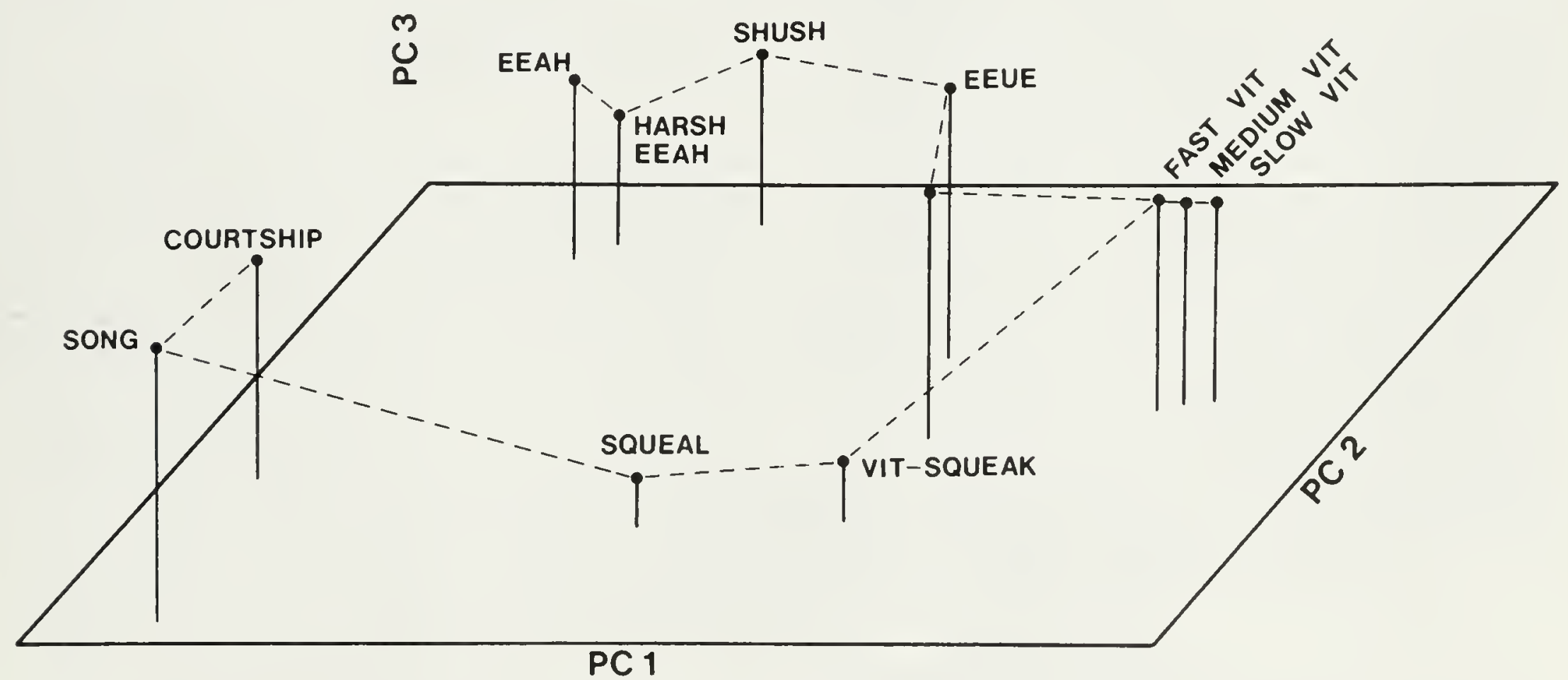

FlG 7. Principal component analysis (PCA) of vocalizations of the Warbling Vireo based on 17 form characters. Lines joining vocalizations represent a shortest spanning tree. 


\section{SIMILARITY COEFFICIENT}

FIG. 8. Distance phenogram from a distance matrix of 17 form characters of vocalizations of the Warbling Vireo. Clustering is by unweighted pair-group averages of standardized characters. The cophenetic correlation coefficient is 0.953 .

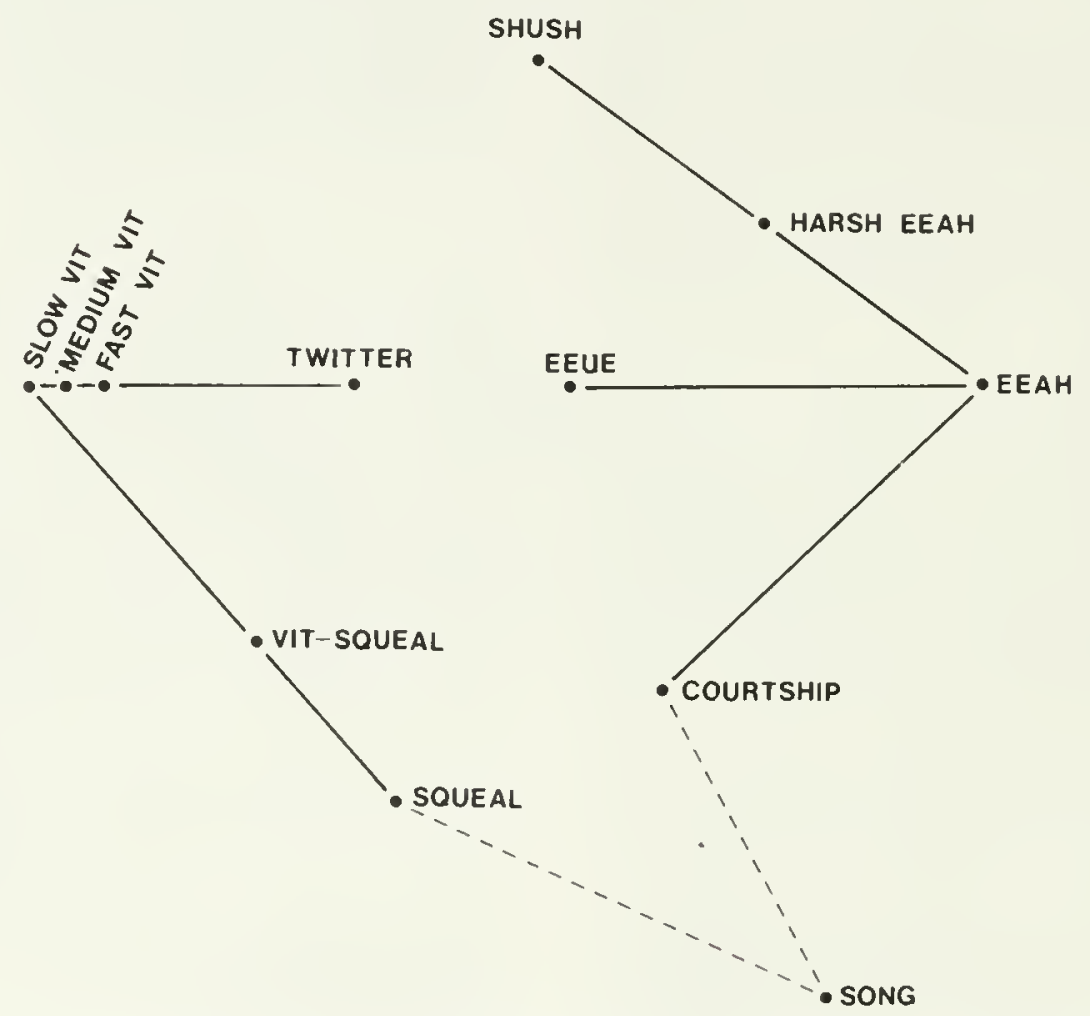

FIG. 9. Schematic representation of form relationships among vocalizations of the Warbling Vireo based on multivariate analysis and qualitative data. Lines join calls that show closest similarity. Dashed lines join song to calls of closest similarity. Proximity on diagram is based on distance measures from multivariate analysis. 
TABLE 4. Form relationships that characterize and differentiate transition series.

Eeah-harsh eeah-shush

-increase in bandwidth

-slight increase in pitch

-figures increasingly similar to noise

-delivery, from one figure to many figures at a time

Eeah-eene

-increase in bandwidth

-decrease in loudness

-decrease in duration of figures

-increase in number of harmonics per figure

-less noiselike

-delivery, from one to many figures at a time

Eeah-partial courtship call-full courtship call

- simple (one figure per call) to complex (many figures per call)

-decrease in duration of figures

-decrease in bandwidth

- decrease in number of harmonics

-figures appear more variable in shape

Slow vit-medium vit-fast vit-twitter

-increase in loudness

-increase in duration of figures

-increase in pitch

-decrease in interval between figures

Slow vit-medium vit-vit-squeak-squeal

-increase in duration of figures

- decrease in interval between figures

-chevron-shaped figures to variable-shaped figures

-increase in lower frequency limit

-decrease in bandwidth

-decrease in number of harmonics per figure

\section{Contextual Relationships}

No obvious trends were apparent in the context category immediate-ongoing-activity (Table 5), except that calls of the vit complex tended to be more associated with flight than those of the eeah complex. In the context category referred to as immediate-environmental-situation (Table 6 ), the proximity of the nest and of interactants appeared to influence calling. Generally, the closer one bird was to another, the greater the probability of calling. Other than during eeahs and courtship calls, the median distance between interactants during calling was less than $3 \mathrm{~m}$. During nesting, a major percentage of eeahs $(63.8 \%, \mathrm{~N}=1223)$, eeues $(100 \%, \mathrm{~N}=12)$, courtship calls $(70.0 \%, \mathrm{~N}=50)$, twitters $(60.3 \%, \mathrm{~N}=68)$, and vit-squeaks $(97.2 \%, \mathrm{~N}=$ 36) were given within $5 \mathrm{~m}$ of the nest. The nest was a natural focal point for all pair interactions.

In the context category referred to as general-environmental-situation (Table 7), different calls were associated with different interactions. In nest defence, eeahs, harsh eeahs, and shushes were common. In territorial defence, eeahs, vits, vit-squeaks, squeals, and twitters were frequently uttered. All calls except shushes were uttered during pair interactions, although eeahs and slow and medium vits dominated.

A principal component analysis was carried out on 28 context characters from Tables 5-7. The three-dimensional PCA ordination values for each call are summarized in Fig. 10. A high proportion of the total character variance $(83.7 \%)$ is explained on the first three principal components, and so the three-dimensional plot is a reliable representation of contextual relationships among calls. Calls cluster in the two call complexes defined in the analysis of form relationships (Fig. 7). The difference is that the eeah shows no close relationship to either group. The eeue clusters with the partial and full courtship calls, and the twitter with the vit-squeak and squeal. Context characters with high eigenvalues on PC 1 are intraspecific interactions. Those with high values on PC 2 are immediateongoing-activity and proximity between interactants. Those with high values on PC 3 are interspecific interactions. Finally, those with high values on PC 4 are sudden or novel stimuli. On PC 1, the eeah is separated from the eeah complex but has affinities with slow and medium vits. Eeahs and vits are separated by distance between interactants and by immediate-environmental-situation context categories.

The results of cluster analyses (Fig. 11) and PCA were comparable. Four major clusters of vocalizations were evident: (1) harsh eeah, shush; (2) eeue, full and partial courtship calls; (3) slow, medium, and fast vits; (4) twitter, vit-squeak, squeal. The eeah showed no close associations with any cluster.

An aspect of context that the multivariate analysis did not reflect was the relative breadth or range of conditions in which calls occurred. To get an estimate of context breadth, the number of situations in which a call was heard was summed up using the multistate values (see bottom of Table 7). Eeah, and slow and medium vits were found in the broadest range of circumstances and in a general set of social interactions (e.g., foraging, trips to and from the nest, and low-intensity social interactions). Henceforth, those calls will be referred to as general-socialvocalizations (GSV's). Calls towards the ends of each of the transition series (Fig. 9) were found in a narrower range of circumstances and in more intense social interactions (i.e., aggression, courtship). Those calls will be termed critical-social-vocalizations (CSV's).

\section{Frequency of Occurrence}

The structure of frequency relationships in a signal repertoire can be examined by ranking signals according to 


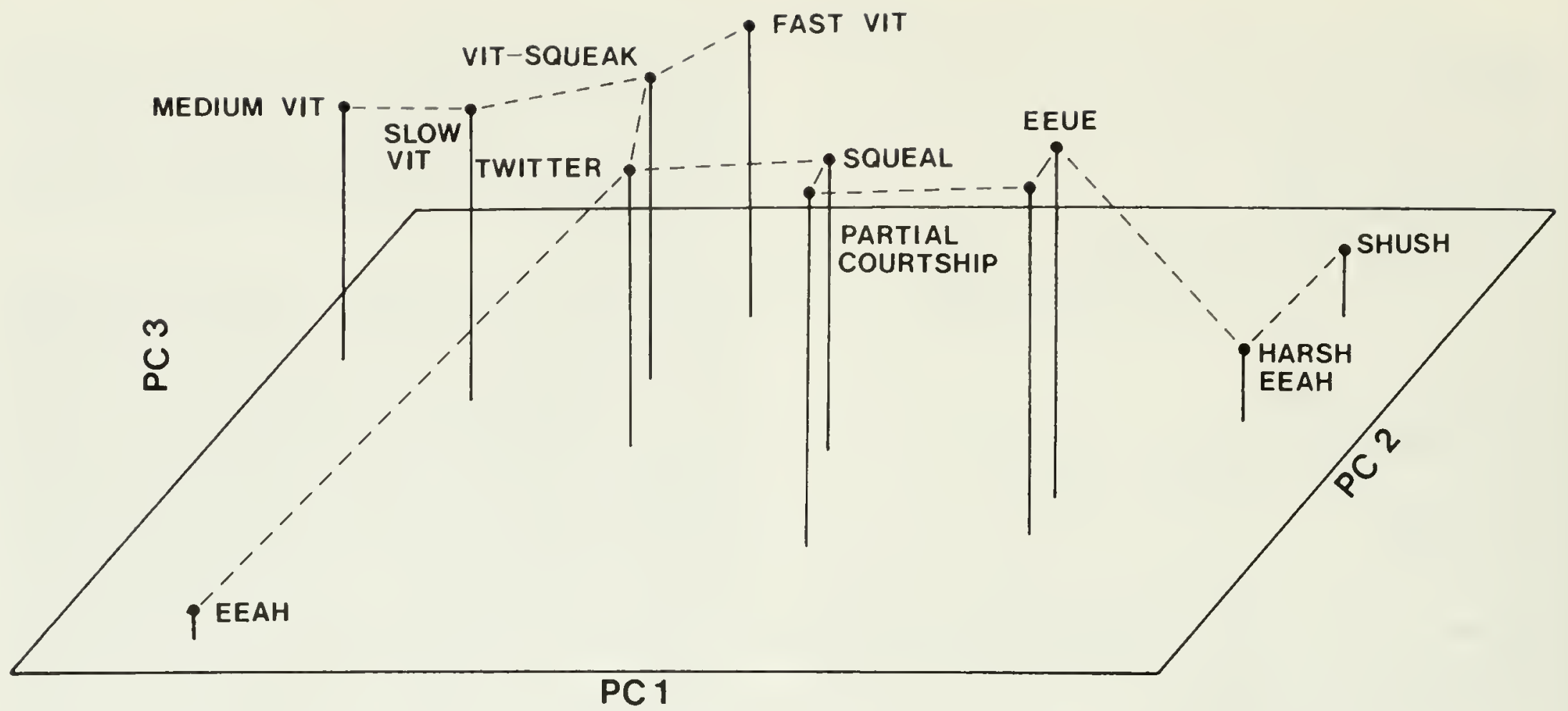

FIG. 10. Principal component analysis of calls of the Warbling Vireo based on 27 contextual characters. Lines joining calls represent a shortest spanning tree.

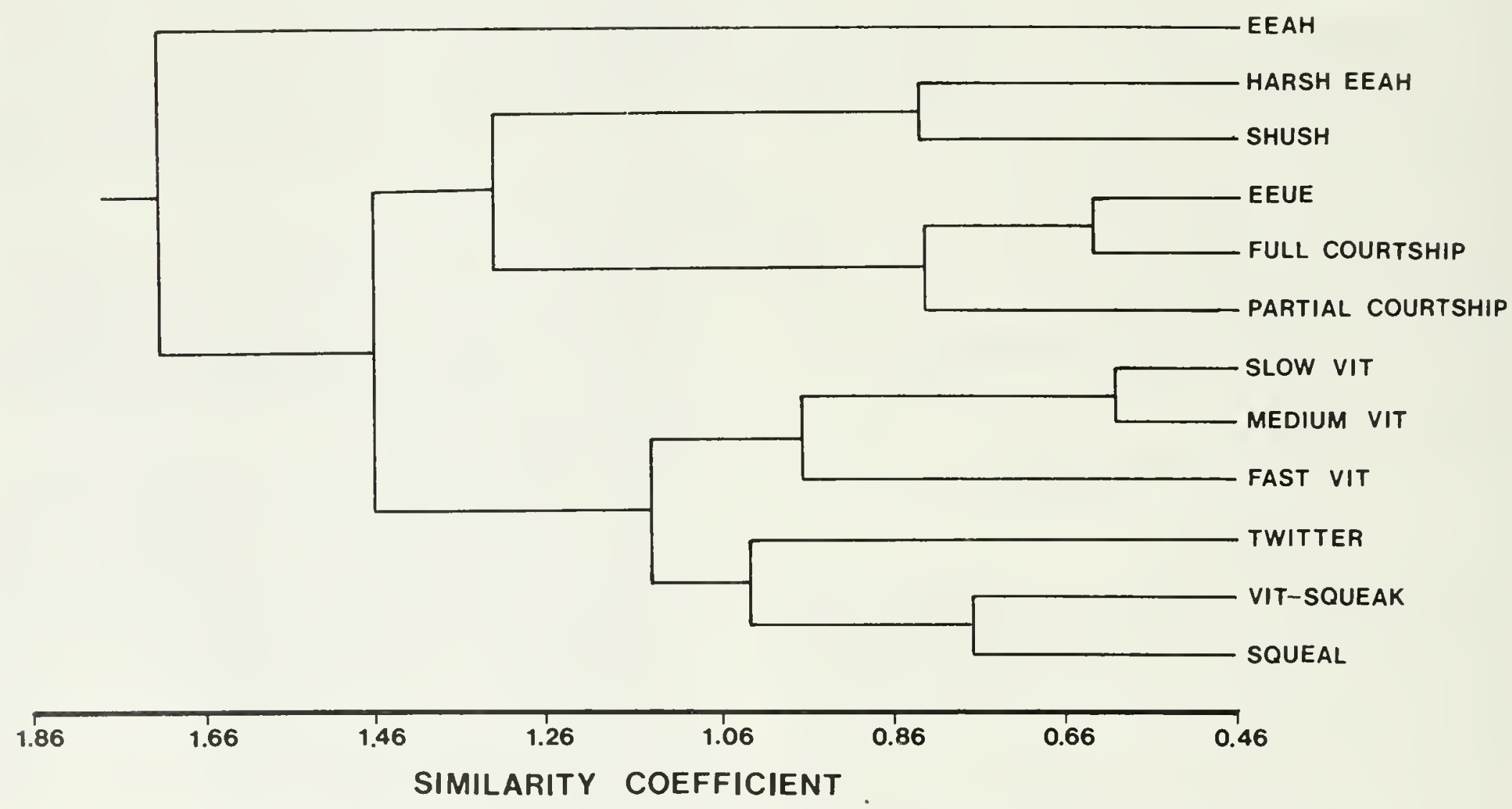

FIG. II. Distance phenogram from a distance matrix of 27 contextual characters of the Warbling Virco. Clustering is by unweighted pair-group averages of standardized characters. The cophenetic correlation coefficient is 0.832 . 


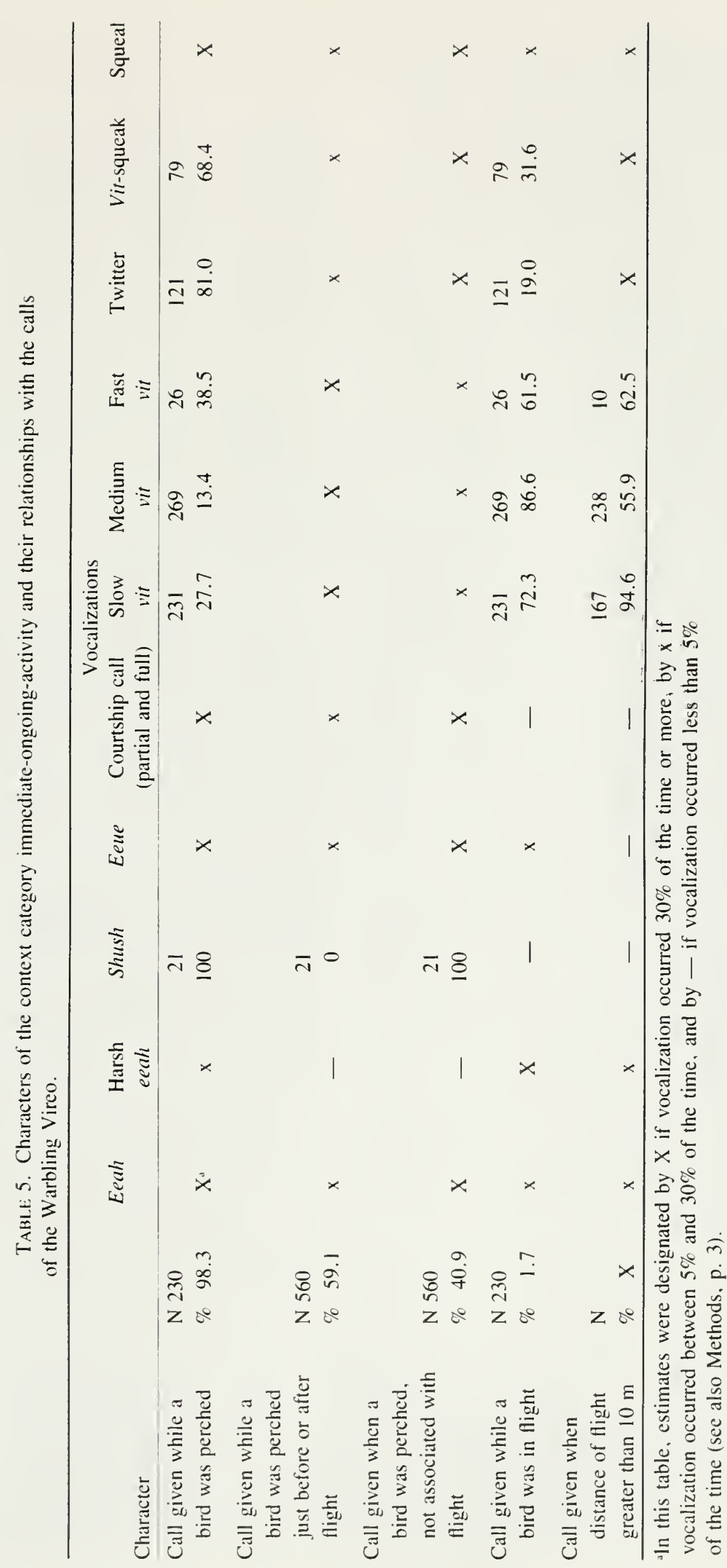




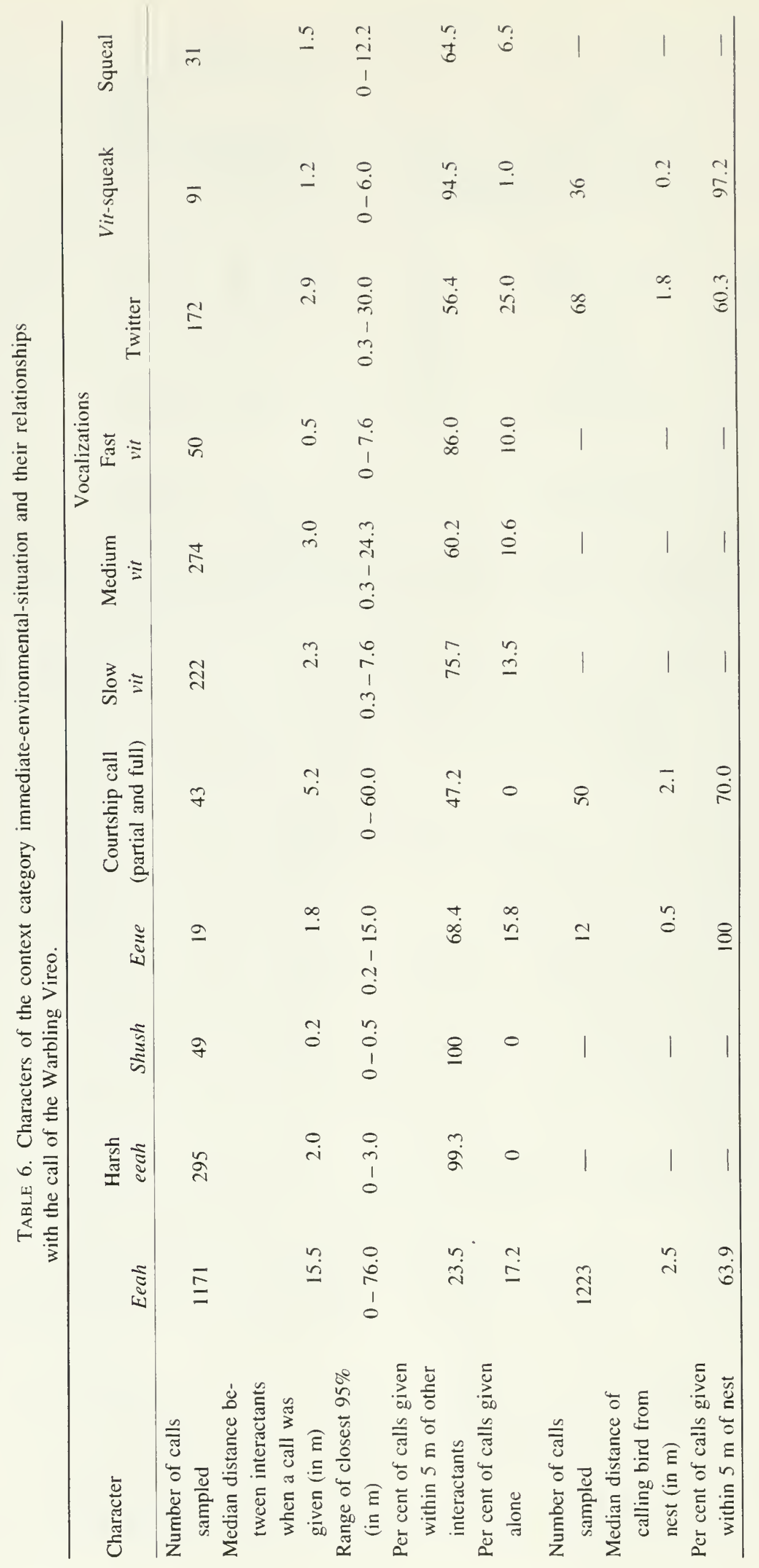




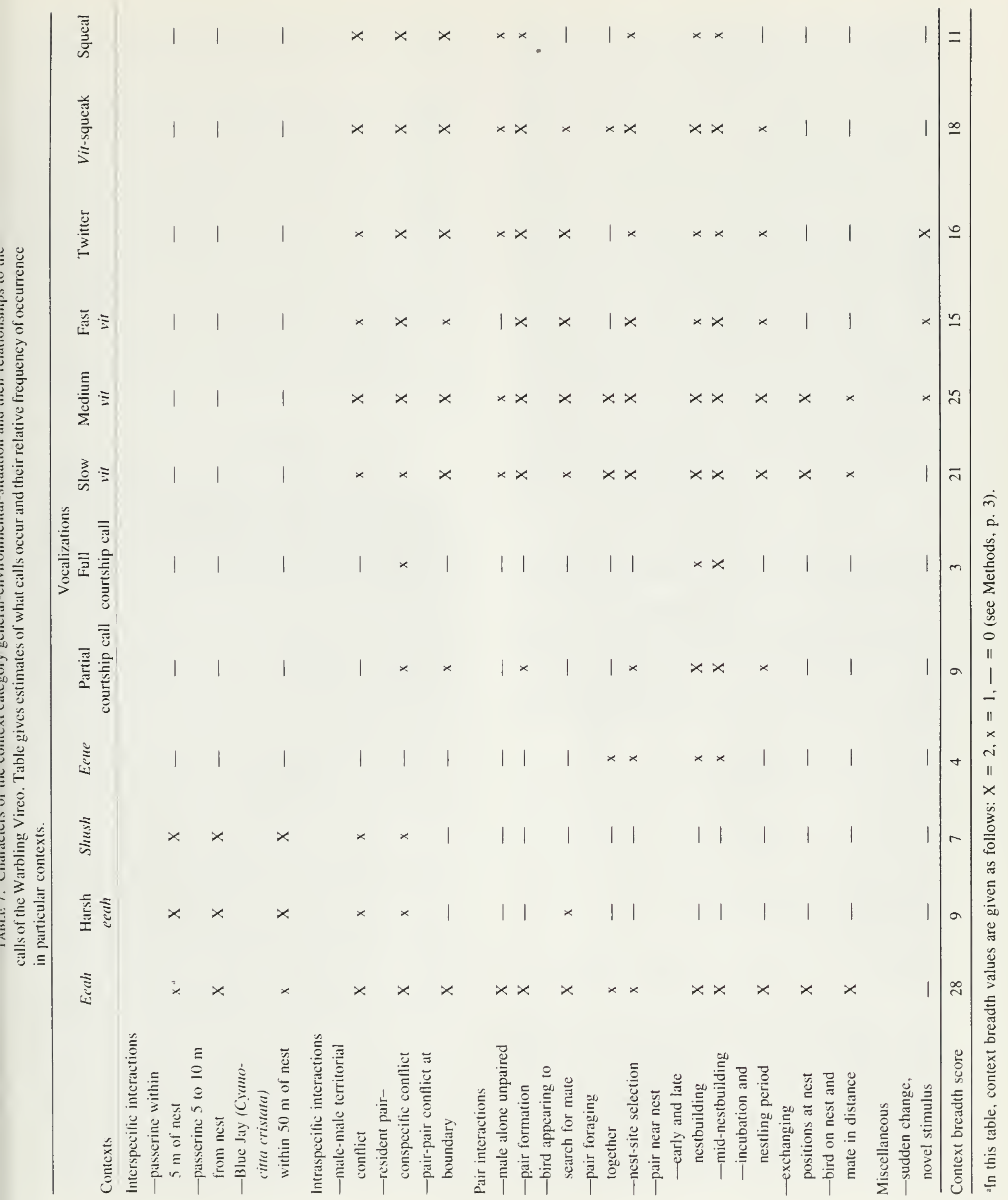




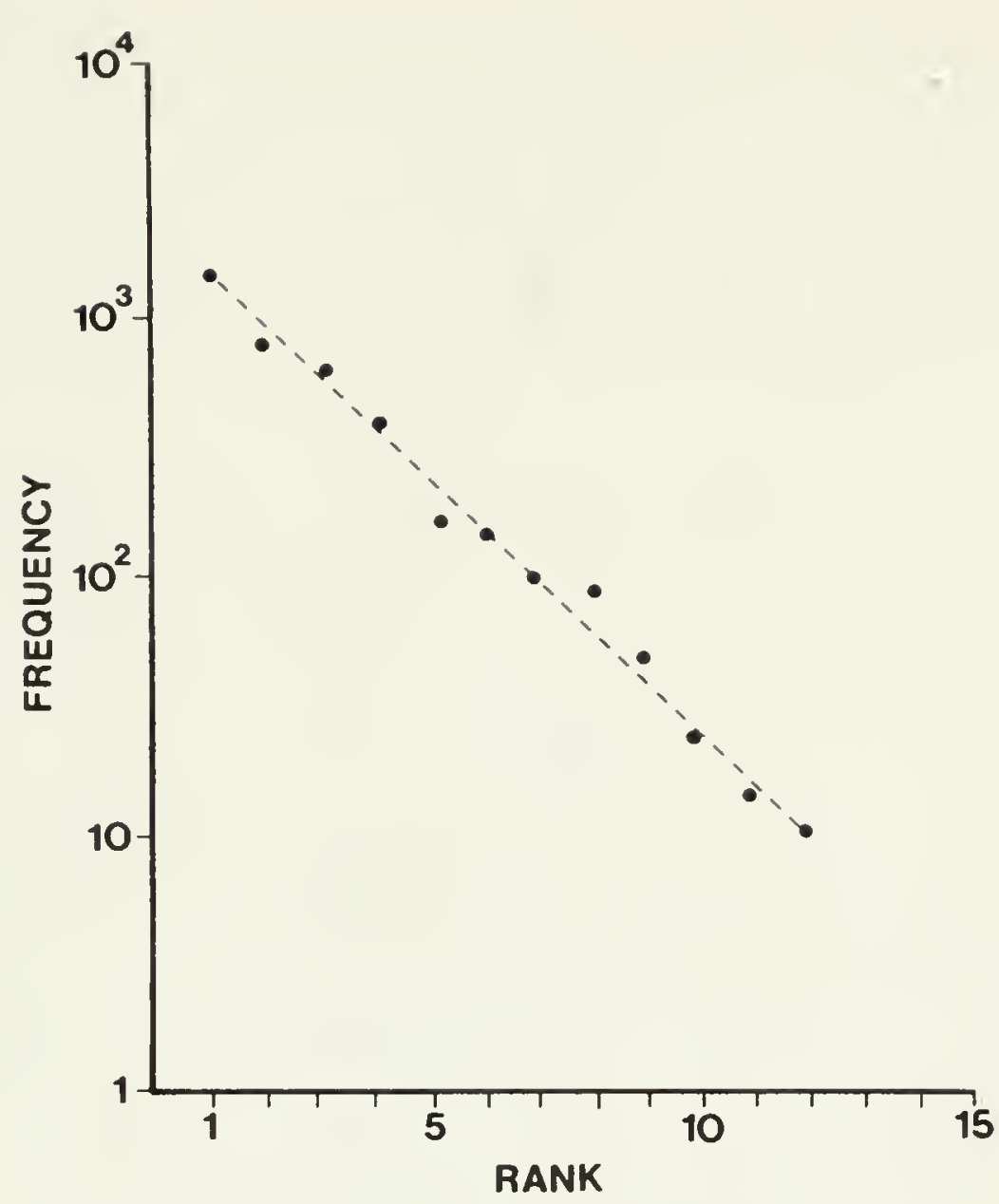

FIG 12. Log frequency of calls versus rank according to frequency of occurrence in the call repertoire (from Table 8).

TABLE 8. Frequency of occurrence of calls of the Warbling Vireo $(\mathrm{N}=375$ calls $)$.

\begin{tabular}{lc}
\hline Call & $\begin{array}{c}\text { Frequency of } \\
\text { occurrence in } \%\end{array}$ \\
\hline Eeah & 36.5 \\
Medium vit & 20.8 \\
Slow vit & 17.0 \\
Vit-squeak & 10.4 \\
Twitter & 4.2 \\
Fast vit & 3.7 \\
Harsh eeah & 2.6 \\
Partial courtship call & 2.4 \\
Squeal & 1.2 \\
Full courtship call & 0.6 \\
Eeue & 0.3 \\
Shush & 0.3 \\
\hline
\end{tabular}

their frequency of occurrence (i.e., the most commonly given signal equals rank 1 , the next most common signal equals rank 2 , etc.) and then plotting them against log frequency. According to Zipf's (1935) law, a negative exponential function should result (Moles, 1963; Schleidt, 1973).

When only calls were considered, the distribution of rank versus log frequency of occurrence for vocalizations

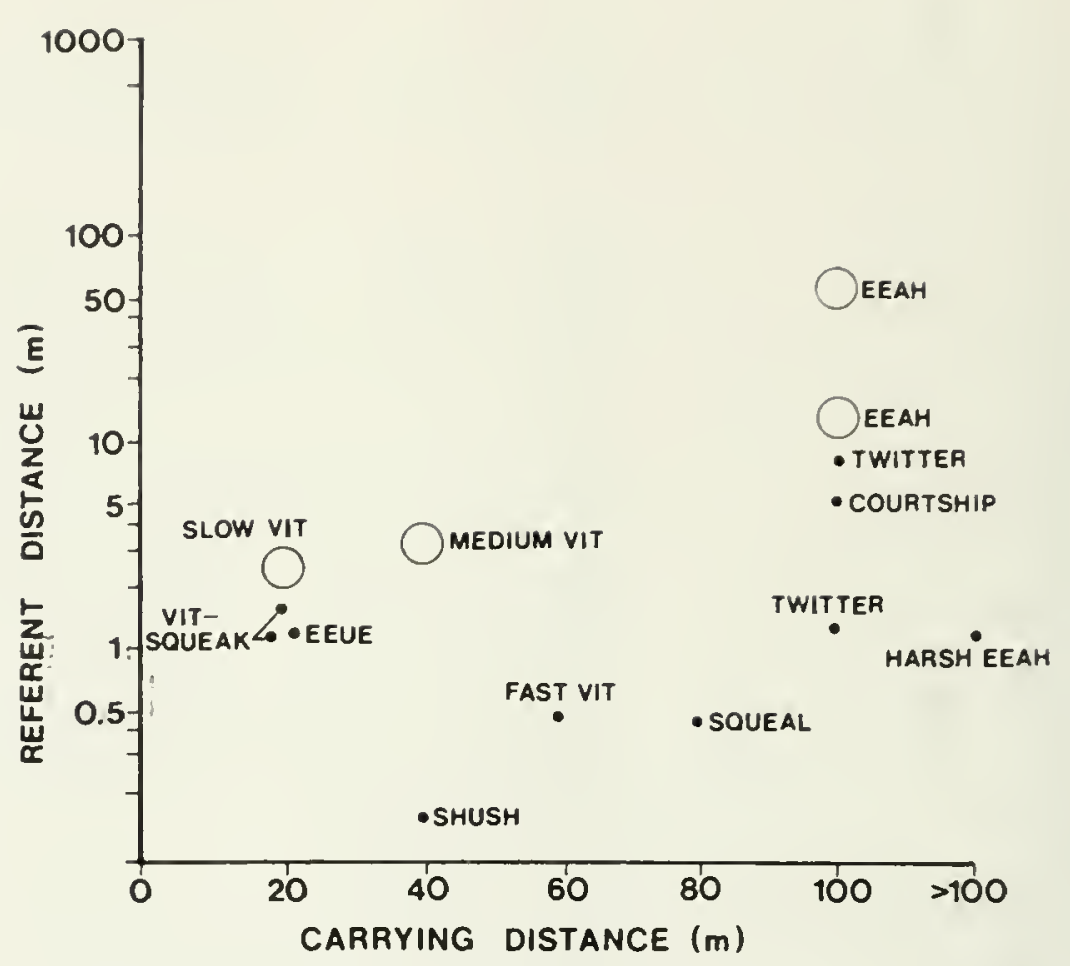

FIG. 13. Summary of relationships between carrying distance and median referent distance. Vocalizations with more than one circle represent samples taken prior to and after the start of nestbuilding. Large circles are GSV's, and small circles are CSV's.

(Table 8, Fig. 13) was close to that predicted by Zipf's law. During the sampling period 28284 songs were counted compared to 3785 calls; however, the method by which song data was gathered (Howes-Jones, 1985a) strongly biased the results in favour of a high frequency of song. Even if an equitable method of sampling frequency were used, song would probably still be more frequent than expected and would lie above the line in Fig. 12.

\section{Loudness and Distance Between Communicants}

Vocalizations such as vits, eeahs, harsh eeahs, vit-squeaks, squeals, and song varied in loudness. Generally. louder renditions occurred in intense social interactions when interactants were close to each other, and quieter versions occurred in general social interactions. The twitter, eeve, and courtship call were more consistent in their loudness.

The carrying distance of vocalizations has been divided into six categories (Fig. 13; see Methods, p. 3). The values do not represent the capabilities of Warbling Vireos, but were expected to be of value in comparing the relative differences in loudness among calls. Several studies (Cohen et al., 1978; Dooling, 1982) have shown that the temporal resolving power of birds is slightly better than that of humans, whereas the intensity-resolving power is slightly poorer. It is likely, therefore, that the estimates of carrying 
distance represented in Fig. 13 are reasonable approximations of the intensity-resolving capabilities of Warbling Vireos.

Most vocalizations between mates occurred during periods when birds were together; for example, $41.6 \%$ $(\mathrm{N}=1171)$ of eeahs, $60.4 \%(\mathrm{~N}=53)$ of courtship calls, and $65.1 \%(\mathrm{~N}=172)$ of twitters were given when birds were within $10 \mathrm{~m}$ of each other. Yet all of these calls were audible up to $100 \mathrm{~m}$ away under good acoustical conditions. This prompted an examination of the relationship between carrying distance and referent distance (median distance between birds when a call was given).

The average of the ratios of carrying to referent distance for the CSV's was 92.1. The value was 11 times greater than the average of the ratios (8.6) for the GSV's. The higher average ratio of the CSV's indicates they were louder than the GSV's with respect to the distance between communicants.

\section{DISCUSSION}

\section{Organization}

The relationships among calls and among the attributes of calls are represented in Fig. 14. Such relationships show that the call repertoire has structure. The presence of structure raises several questions. What constraints are responsible for the particular design of the call repertoires? How does the organization of the structure influence communication between Warbling Vireos? Is the organization unique to the Warbling Vireo, or is it also relevant to other systems of avian vocal communication? What insight does structure provide into the possible evolution of the call note system?

\section{Message and Context}

A major constraint acting on animals is the limited capacity of their information-processing systems (Fentress, 1976; Barlow, 1977). This constraint is general to all open living systems (Miller, 1978:121-202). Limited capacity influences the design of animal communication systems by restricting an animal to a circumscribed number of displays (see Moynihan, 1970; Smith, 1977). This restriction forces displays to be dependent on contextual sources of information for their interpretation. The importance of context in interpretation of animal displays has been well established (Armstrong, 1973; Smith, 1977; Lein. 1980; Catchpole, 1982).

Johnston (1976) argues that small signal sets maximize the likelihood of clear, equivocation-free transmission of signals. Similarly, Smith (1977) suggests that a limited repertoire results from the inability of an individual to be perceptually ready for rare displays and the retarded response rate to large sets of stimuli. A limited number of displays restricts the "message space." This in turn imposes constraints on the message categories (Smith, 1977). Relationships between context and message have been discussed in detail by Smith (1977); however, his ideas have seldom been applied by others to studies of display repertoires. The results of this study support his observations.

According to Smith (1977), selection favours that both narrowly and broadly predictive behavioural messages exist in some balance in the display repertoire. In the Warbling Vireo, a balance is evident, but broadly predictive messages predominate. Broadly predictive messages (i.e., general-social-vocalizations) are imprecise and can be given in many situations; such messages contribute to the selection of a large number of differing responses from recipients and as a consequence are heavily dependent on contextual sources of information for their interpretation. Narrowly predictive messages (i.e., critical-social-vocalizations) are more precise, are performed in fewer situations, and are less dependent on contextual sources of information for interpretation. The constraints imposed on context and message relationships also extend to the frequency of occurrence of calls.
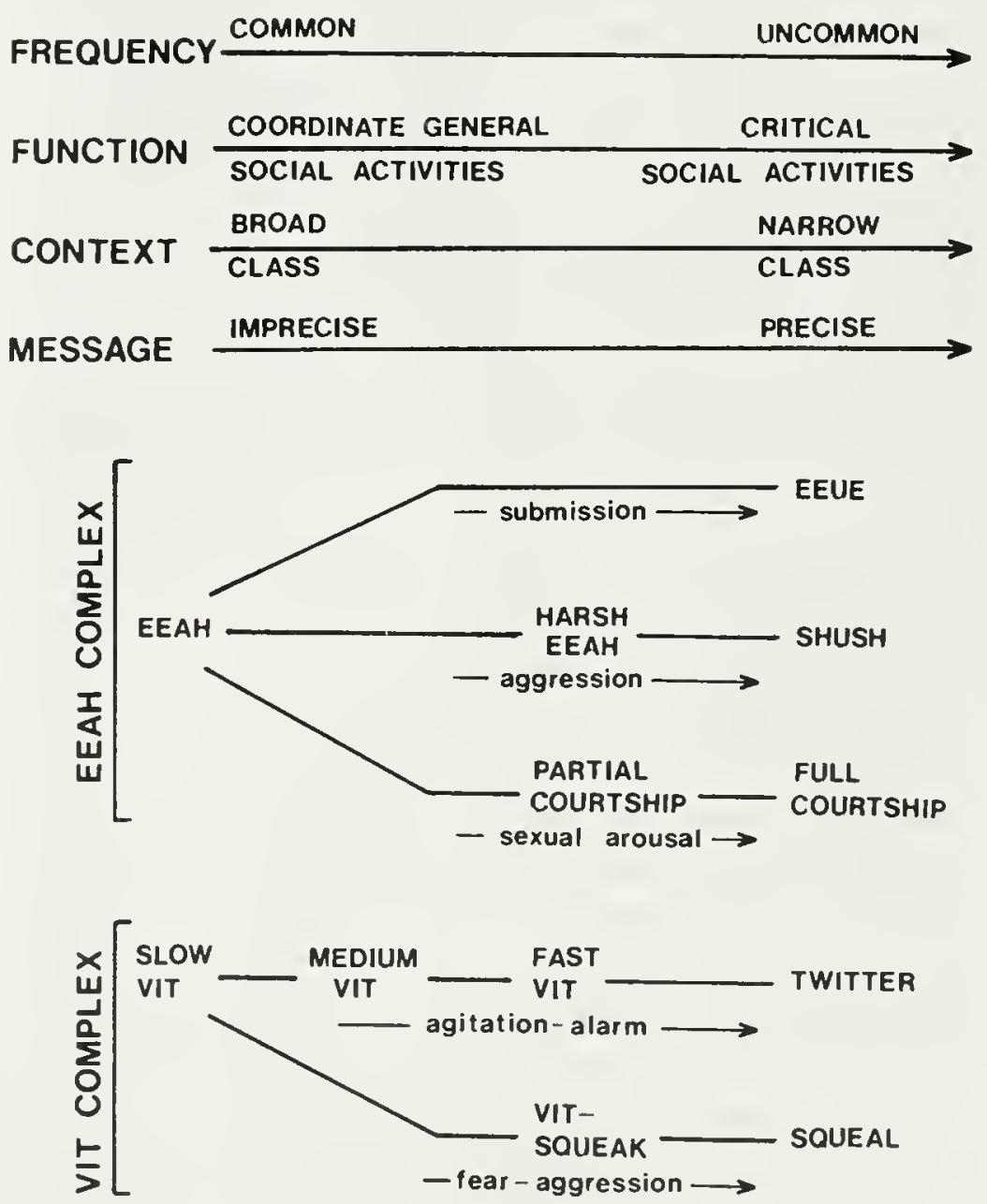

FIG. 14. Schematic representation summarizing relationships among vocalizations of the Warbling Vireo in form, message, context, frequency of occurrence, function, and presumed motivation. Solid lines join calls that grade in form or show closest form relationships. 


\section{Frequency of Occurrence}

The frequency of occurrence of Warbling Vireo calls approximates Zipf's (1935) law (Fig. 13). Zipf (1935; see Moles, 1963) suggested there exists a constant dialectic equilibrium between two contradictory tendencies in the receiver and source individuals. One is the social tendency that demands a precise and complex message leading to a highly varied language, whereas the other tendency is towards brevity or least effort in the message.

Two factors may account for the frequency relationships found in Warbling Vireos. First is the association between frequency of occurrence and variation in context breadth. The product-moment correlation coefficient between context breadth (Table 7) and frequency of occurrence of calls (Table 8$)$ is significant $\left(0.90^{* *}, N=12\right)$. If it is assumed that the elicitation of a vocalization is contingent upon a set of configurations of causal factors or variables (McFarland and Sibley, 1975), then it can be assumed that frequency of occurrence will depend on how many times in the course of a vireo's activities these configurations of variables occur. A call associated with a large set of configurations is expected to have a wide context breadth. If the requisite configuration of conditions repeatedly arises, then the call associated with those conditions will also be frequent. For example, squeals were most often given when two or more males were engaged in a territorial dispute. In isolated territories (few neighbours), the number of interactions between males was concomitantly reduced and the number of squeals was also reduced. Where territories were more densely packed, interactions were more frequent with a concomitant increase in the number of squeals.

A second factor that likely influenced the frequency of occurrence of calls was function. It has been suggested (Schleidt, 1973; Heiligenberg, 1974) that a single discrete signal has an effect on the receiving animal that waxes and wanes as time progresses. If the signal is repeated, its effect may add to the residual effects of previously received signals and yield a cumulative or tonic effect in the receiving animal. These effects have been variously described as being "priming" (Wilson, 1971:234), or "stimulative" and "informative" (Huxley, 1923; see Smith, 1977:266). Since individuals must contend with a wide range of social situations, both common signals (low information-tonic effect) and rare signals (high information-discrete effect) are selected for the repertoire (see Smith, 1977:173). Common signals are of value in maintaining and integrating general social relationships or in priming the mate for copulation (Hinde, 1965; Lehrman, 1965; Brockway, 1969; Wiley, 1976b; Silcox and Evans, 1982). Rare signals are of value for their startle effect or where an immediate response is required of the recipient, as in the mobbing of a predator or in territorial interactions (Andrew, 1964; Moynihan, 1970).

\section{Distance Between Communicants}

The design of a vocal communication system is influenced by the distances over which communicants are signalling (Marler, 1968; Wilcy, 1976a, 1976b, 1976c) in conjunction with properties of their environment (Morton, 1975; Nottebohm, 1975; Marten and Marler, 1977). In birds, the relationship between the effects with respect to distance of environmental factors on acoustic variables has been well established. However, most data on distances between interactants is qualitative. Among primates, signals exchanged over a distance are louder and more stereotyped, whereas communicants that are in close proximity communicate by graded signals (Marler, 1976a). The latter relationship also appears to hold for the Warbling Vireo.

In most monogamous territorial passerines, as in the Warbling Vireo, most calls are probably directed at mates (see Lein, 1980). Therefore, the design of a call note system might be adapted to the range of distances usual to mates. During the breeding season, mated Warbling Vireos spent about $75 \%$ of their time more than $10 \mathrm{~m}$ apart (Howes-Jones, 1985b). Despite this, most vocalizations were given when birds were within $10 \mathrm{~m}$ of each other. Barlow (1977; see also Hartshorne, 1973) suggested that in pair interactions, mates become accustomed to one another; this leads to selection for variation and novelty in signals in order to maintain responsiveness and attention. Members of a social group that are familiar with each other (i.e., in constant proximity) tend to have graded signals, while signals between unfamiliar individuals are more stereotyped (Barlow, 1977).

Schleidt (1973) divided the effective range of a signal into "two concentric sections, namely a core area in which the signal can be received over its full range of intensities and a surrounding ring-shaped periphery in which noise gnaws away the lower intensity range." The area in which the receiver can use the full range of intensities will be only part of the total area in which a signal can be heard when emitted with maximum intensity. In this study, the high ratio $(69.3)$ of the carrying distance to the referent distance (i.e., calls appear louder than required for proper reception with respect to the median distance found between communicants) tends to support Schleidt's hypothesis. The high ratio also suggests that the volume of the signal has evolved so that it is loud enough to be effective in the few circumstances in which the signal is given when birds are far apart. Confining vocal communication to periods when birds are close together reduces the signal-tonoise ratio and allows for the simultaneous reception of vocal and optical displays, thus reducing ambiguity of message (Marler, 1961, 1967; Smith, 1969). Apart from its having advantages of signal reception, proximity, as suggested by Marler (1976b), is the most general and basic stimulus provoking aggression in animals. Since close proximity is essential for most pair activities, selection 
might favour close-range conmunication that allows interactants to monitor each other.

In some contexts, especially those involving aggression, the high volune of an unexpected call (i.e., harsh eeah) may surprise or startle a recipient (Moles, 1963; Schleidt, 1973). Animals generally approach stimulation of moderate intensity and withdraw from intense stimulation (Andrew, 1964). The lower ratio of carrying-to-referent distance for general-social-vocalizations may be due to the fact that those vocalizations are common and may attract the attention of predators.

\section{Form and Motivation}

It is likely that all the calls of the Warbling Vireo are influenced by the same set of causal factors. In any instance, causal factors are present that may influence the elicitation of many calls. The call given, however, depends on the particular configuration or combination of causal factors (see McFarland and Sibley, 1975). The external causal factors that appear most important to Warbling Vireos are (1) proximity of interactants to each other and the nest, (2) the degree of familiarity, and (3) the time of year. Marler (1976b) considered proximity and degree of familiarity as the most important factors underlying aggression. Their importance in the social relationships of birds has been well established (Erickson. 1973; Caryl, 1975; Silcox and Evans, 1982). In this study, the calls of each transition series appeared to be associated with a similar set of causal factors. To some extent, changes in form along each transition series are typical of those associated with changes in stimulus contrast (Andrew, 1961a, 1964). The call given appears to depend on the intensity of the contrast, with the intensity being primarily determined by the proximity and persistence of the stimulus.

Changes in form in each transition series, and the behaviour of birds associated with those changes, follow closely the predictions of Morton's (1977, 1982) motivational-structural rules. The eech-to-shush transition series has the structure and harsh sound characteristic of mobbing calls of most passerines, including other vireos (Marler, 1955; Curio, 1975; Morton, 1975; Barlow and Rice, 1977). The eelue shows an opposite trend and is associated with a nonaggressive state.

Certain characteristics of the courtship call (combination of figures that are tonal and harsh, rapid fluctuations in sound frequency) indicate conflicting motivation and are typical of precopulatory solicitation signals of many passerines (Andrew, 1957, 1961b). Morton (1977) suggested that the chevron-shaped characteristic (e.g., vit and twitter) indicates conflict or approach-avoidance behaviour. The higher frequency and greater intensity of the twitter may indicate heightened fear (Morton, 1977).

\section{Structure and Interpretation}

In the Warbling Vireo, the form of calls may be described as a series of modal peaks along continua of change (see Jenni et al., 1974), with the peaks approximating typical intensity-type calls (Morris, 1957). The calls that comprise parts of a transition series, such as fast vit-twitter, vit-vitsqueak, eeah-courtship call, have more distinct boundaries between them or show a small abrupt change in form, while calls such as slow-medium-fast vits and eeah-harsh eeah show continuous change. Song is the only vocalization in the repertoire of the Warbling Vireo achieving such a degree of complexity that a differentiation of form has occurred within its structural framework and on a number of levels (Howes-Jones, 1982, 1985c).

Animal displays are usually categorized as discrete (digital) or graded (analogue) (Marler, 1961; Konishi, 1963; Sebeok, 1965). The adaptive advantages of both types have been widely discussed in the literature (Marler, 1961, 1965, 1967, 1976a; Konishi, 1963; Sebeok, 1965; Smith, 1969; Schleidt, 1973; Wilson, 1975; Barlow, 1977). These categories have often been applied in an either-or fashion. As a result, vocal repertoires have often been portrayed as aggregates of nonoverlapping vocal types. Pattern and variation are not mutually exclusive and both can be achieved in motor output (Wiley, 1975; Barlow, 1977). In other words, degrees of discreteness or gradedness can be expected in displays.

Degrees of relationship have been reported among calls of birds; however, most studies have been qualitative, restricted to one or two attributes, or have involved only a specific group of calls (Marler, 1956; Andrew, 1961a; Konishi, 1963; Smith, 1966, 1970a, 1970b; Oring, 1968; Thompson and Rice, 1970; Wiley, 1975; Wilkinson and Howse, 1975; Thompson, 1976; Zann, 1977; Ficken et al., 1978; Hardy, 1979; Lein, 1980). Recent studies have shown that avian vocal repertoires may be more graded and organized in a more complex manner than previously thought. For example, Huxley and Wilkinson (1977) found that the calls of adult Aldabra White-throated Rails (Dryolimnas cuvieri aldabranus) fell into two groups of interrelated calls, one representing alarm and interspecific aggression and the other, sexual and social contexts. Jenni et al. (1974) divided the vocalizations of the Northern Jacana (Jacana spinosa) into six classes, five of which belonged to a single graded series.

A consequence of having an intergraded call repertoire is that the message of any call may be misinterpreted by the recipient. Qualities useful in preventing misinterpretation include high stereotypy and redundancy (Morris, 1957; Marler, 1961, 1967; Smith, 1969). In the Warbling Vireo, several factors may operate to reduce message ambiguity. Vocalizations that tend to occur together belong to different complexes or different transition series. For example, in most general-social-interactions, eeahs, slow 
to medium vits, and song are uttered. Message misinterpretation could also occur between call forms along the same transition series or between general-social-vocalization variants that sound similar (i.e., distorted eeahs, eeue-like eeahs, and loud eeahs). Compatibility between a variant message and the original can be achieved if the failure by the recipient bird to differentiate between the two variants is not significant to the recipient (Smith, 1969). The messages of general-social-vocalizations tend to be imprecise enough that misinterpretation of a variant form would probably be of little consequence. In the case of related forms in a transition series, calls have similar message and contextual properties.

In a theoretical analysis based on semiotic processes, Johnston (1976) argued that the tolerance space of a legisign (established rule between sign and object) is fuzzy. This fuzziness is of importance in communication systems since it allows the legisign associated with a particular semiosis to be gradually shifted in some direction without causing a breakdown in the coherence of the semiosis. Since most critical-social-vocalizations are uttered when interactants are in close proximity, a continuously variable signal may convey subtle changes in motivational information that may be important to the outcome of the interaction (i.e., aggression [Marler, 1961]).

In critical-social-interactions (e.g., courtship, aggression) it is important that the message be conveyed unambiguously to the intended recipient (Marler, 1956, 1961; Barlow, 1977). Calls used in these interactions tend to be the ones at the ends of the transition series. These calls are dissimilar, loud, and given close to other interactants, and so it is unlikely that they will be misinterpreted. Compared to general-social-vocalizations, they are also more redundant. For example, harsh eeahs and squeals are generally repeated until the termination of the interaction. Courtship calls and twitters, although not often repeated, consist of a number of figures. Rare signals must be stereotyped so that a receiver can detect and recognize them (Marler, 1956, 1961; Barlow, 1977). This relationship appears to hold for harsh eeahs, eeues, and twitters, but not for squeals or courtship calls, which are variable. However, because these latter two calls are distinct and repeated, stereotypy may not be important for their interpretation. In courtship and aggressive interactions, some variation may be advantageous to prevent habituation (Hartshorne, 1956; Kroodsma, 1978). It has usually been assumed that, by using graded signals, a signaller benefits by conveying its precise motivational state to others (Smith, 1977). However, Dawkins and Krebs (1978) argued that contestants in ritualized confrontation should conceal their exact motivational state and display signals with typical intensity. Why the latter argument does not often hold, as in this study, probably relates to the fact that calls often function in many, and often somewhat unrelated, contexts that may demand compromise in the distinctiveness of their form.

\section{Structure and Observer Interpretation}

The manner in which observers organize their precepts can influence how they eventually interpret the organization of a system. One such process is the tendency to categorize and the failure to realize its limitations. The assignment of categories to perceptions is in part the heritage of the cognitive structuring character of our Indo-European language systems (Bertlanffy, 1962; Rapoport, 1978). The problem applies to the interpretation of avian vocal repertoires (i.e., Jenni et al., 1974). Huxley and Wilkinson (1977) have shown that classifications of signals based on short-term studies and with qualitative evidence may be oversimplified and mask underlying relationships. For example, Andrew (1964) found the calls of chicks of domestic fowl to represent a continuous series of patterns, whereas in a less detailed study, Collias and Joos (1953) described four distinct calls for chicks. After the first field season, my impression was that most calls were distinct. This resulted from the fact that calls that occurred together in a given situation were aurally distinct, giving the impression of a repertoire of distinct call types.

\section{Self-organizing and Interactive Character of the Call Note System}

Fentress (1973, 1976) argued that behavioural systems are both interactive and self-organizing and that the balance between these two modes of organization can shift as a function of dynamic principles. In other words, the system redefines its control boundaries as a function of its state of activation. The call note system of the Warbling Vireo shows similar characteristics. At low levels of information input or at relatively low levels of activation (defined as the degree of disturbance, arousal, attentional demands. stimulus strength), a behavioural system produces relatively diffuse excitatory effects on motor outputs. The result is that a wide range of behaviours can potentially be produced (Fentress, 1973, 1976). As examples, eeahs and slow and medium vits are given when birds appear in a state of low or moderate activation. Why a large number of calls is not produced relates to the fact that the number of signals and messages available to Warbling Vireos is limited, as suggested by Fentress. The problem of limited message space can be resolved if fewer calls function in a greater number of contexts with a consequent increase in their frequency of occurrence.

With an increase in the level of activation, the inhibitory boundaries of a given system tend to expand. or the behavioural system becomes increasingly isolated from the influence of external factors. Behaviours that normally 
would be given at low levels of activation are now blocked. At higher levels of activation, the range of behavioural outputs that are facilitated becomes smaller, with the result that there is an increased likelihood of specific and stereotyped behaviours (Fentress, 1976). In the Warbling Vireo, these changes correspond to calls produced towards the ends of the transition series. Such calls tend to be redundant or stereotyped (see Structure and Interpretation, p. 27) and function in specific contexts. Since the Fentress model is general in scope, it is anticipated that the call note systems of other birds may be similarly organized (see Fig. 14). Huxley and Wilkinson (1977) found that calls of the Aldabra White-throated Rail could be arranged in continua radiating from central calls that were associated with the state of arousal. In general, comparisons are few because the question of organization in vocal repertoires has largely been ignored in other studies.

\section{Evolution of the Call Note System}

The information generated by the analysis of relationships (see Fig. 14) provides insight into the possible evolution of the call note system. It is suggested that eeah and vit are ancestral to, and precursors of, the calls in the transition series. Evidence in support of this hypothesis is as follows: (1) Eeahs and vits would be resistant to evolutionary change because their generalized messages would be preadapted to a wide range of social settings; calls along the transition series have more specialized messages and would likely be more influenced by changes in the environment or in social context. (2) Assuming that vocalizations evolve by a process of progressive differentiation from ancestral forms (Moynihan, 1970), the gradation of form, context, and functional relationships (see Fig. 14) occurring along each of the transition series suggests development along the paths in the direction of that gradation. (3) The calls that are most similar in form, function, and context in other vireosylvids (Philadelphia and Red-eyed vireos) are the eeah-like and vit-like calls (Lawrence, 1953; Barlow and
Rice, 1977). (4) The calls of the young resemble the vit and eeah calls, and the first adult calls to develop are the eeah and vit (Howes-Jones, 1984).

Moynihan (1970) suggested that when a signal occurs frequently, its message becomes increasingly meaningless. This can be avoided if the display is constantly reinforced by new variations or elaboration, with the direction of change being determined by its effectiveness in eliciting a response from other individuals (Tinbergen, 1952). In this study, the transition series are examples of how such variation or elaboration may manifest itself.

It is generally accepted that song evolved from call notes; however, the literature is scanty (Lanyon, 1960; Andrew, 1961b; Armstrong, 1973). The analysis of relationships (see Figs. 8,9) suggests that Warbling Vireo song originated from the eeah complex and specifically from a courtshiplike call. Several lines of evidence suggest this conclusion. Like eeahs, song is used to coordinate nest exchanges and appears to sexually stimulate the female during the nestbuilding period (Howes-Jones, 1985a). The frequency of occurrence of song and courtship calls is highest near the nest where pairs often engage in loosely synchronized duets of courtship calls, song, and eeahs. The differences between the courtship calls and song vocalizations are that song is given more frequently and fulfils a wider range of functions. Song has likely undergone a rapid differentiation and elaboration of form as a result of its territorial, sexual, and general social functions (Armstrong, 1973; Krebs, 1977; Catchpole, 1982). Displays associated with reproduction generally undergo rapid evolutionary change to facilitate mate recognition (Hinde, 1955/1956).

The gradation of song figures with squeals may represent a motivational continuum of probabilities of escape and aggression. High-pitched and distorted figures are common to the calls and song of many passerines and represent similar motivation among species (Armstrong, 1973; Morton, 1977). The association of squeals and song has likely occurred secondary to the development of song. 


\section{Summary and Conclusions}

The vocal repertoire of the adult Warbling Vireo consists of 12 calls and male song. Analysis of form relationships showed the call repertoire to be comprised of two complexes: eeah and vit, each consisting of two and three graded series, which appear to grade from eeah and vit calls (Fig. 9). Changes along each transition series appear associated with changes in stimulus contrast. Each series appears associated with a different behavioural tendency, and the changes in form along each series correspond closely with those predicted by Morton's $(1977,1982)$ motivational-structural rules. Most calls were exchanged when mates were in close proximity; this may account for the gradations in the call repertoire. Calls were described as modal peaks along continua of change. Potential problems in the interpretation of calls by conspecifics could be obviated by the manner in which form relationships are organized and in which combinations of signals are used together in different contexts.

The results of the analysis of contextual relationships (Figs. 10, 11) were similar to those of form. Two groups of calls were distinguished: general-social-vocalizations and critical-social-vocalizations. The former calls were associated with low-intensity social interactions, functioned primarily in the coordination of movements, and facilitated the establishment and maintenance of the pair-bond. With respect to form, the eeah and vit were located at the hubs of each call complex (Fig. 14). The latter calls functioned in more intense and agonistic interactions.

Most calls appeared to be dependent upon contextual sources of information for their interpretation; however, there was a range of relationships. The pattern in the frequency of occurrence of calls corresponded to Zipf's law. General-social-vocalizations tended to occur in a broader class of contexts, had less precise messages, were more frequent in occurrence, and were less loud with respect to distances between interactants than critical-social-vocalizations (Figs. 12-14).

General-social-vocalizations were suggested to be more primitive and resilient to change, whereas critical-socialvocalizations were more recent and subject to change. Song may have evolved from a call resembling the courtship call of the female.

Context, message, and frequency of occurrence were interdependent. and relationships between these were related to limitations in information-processing capacity and the message space available to animals. The manner of organization appears to maximize the functional use of calls even though the number of calls and the messages conveyed by those calls are limited. The organization also relates message and function to form changes in a manner that likely minimizes interpretation errors.

The call note repertoire of the Warbling Vireo has structure and organization and can be considered a system rather than an aggregate of calls. It appears to be interactive and self-organizing, conforming to a general behavioural model proposed by Fentress (1976). The question of organization in avian call repertoires has not been well addressed. Representations of call repertoires may be over-simplified and subject to perceptual biases of the investigator.

\section{Acknowledgements}

This study was carried out as part of doctoral research by Howes-Jones at the Department of Zoology, University of Toronto, Toronto, Canada. We wish to express our sincere thanks for the suggestions and constructive criticisms offered by Dr. G. Morris, Dr. W. Thompson, and several anonymous reviewers. We are grateful to Heather Ardies and Margaret Goldsmith for help in processing this paper.
We are indebted for the financial support provided by the National Science and Research Council of Canada grant A-3472 to Dr. J. C. Barlow. We would like to acknowledge the generosity of the management of the Royal Botanical Gardens, Hamilton, Ontario, for maintaining Cootes Paradise Sanctuary as a natural habitat for the public good and for its availability for scientific research. 


\section{Appendices}

Appendix 1. Characters used to describe form relationships among vocalizations of the Warbling Vireo, and the coding methods for multivariate analysis. Data on song from Howes-Jones (1982).

\begin{tabular}{|c|c|}
\hline Character & Coding and Description \\
\hline Number of figures & $\begin{array}{l}0 \text { given one at a time } \\
1 \text { given singly or a number at a time }\end{array}$ \\
\hline Intervals between figures & $\begin{array}{ll}0 & \text { duration variable } \\
1 & \text { duration regular }\end{array}$ \\
\hline Figures per bout & $\begin{array}{l}0 \text { figures given in bouts } \\
1 \text { bout structure not developed, figures given a few at a time or continuous }\end{array}$ \\
\hline Duration of figures & from Table 1 \\
\hline Loudness (in terms of distance) & $\begin{array}{ll}1 & 0-20 \mathrm{~m} \\
2 & 21-40 \mathrm{~m} \\
3 & 41-60 \mathrm{~m} \\
4 & 61-80 \mathrm{~m} \\
5 & 81-100 \mathrm{~m} \\
6 & \text { greater than } 100 \mathrm{~m}\end{array}$ \\
\hline Metallic & $\begin{array}{l}0 \text { nonmetallic sound } \\
1 \text { metallic sound }\end{array}$ \\
\hline Squeal & $\begin{array}{ll}0 & \text { non-squeal sound } \\
1 & \text { squeal sound }\end{array}$ \\
\hline Nasal & $\begin{array}{l}0 \text { non-nasal sound } \\
1 \text { nasal sound }\end{array}$ \\
\hline Whistled & $\begin{array}{ll}0 & \text { non-whistled sound } \\
1 & \text { whistled sound }\end{array}$ \\
\hline Number of bands in figures & $\begin{array}{l}0 \text { fewer than three or three distinguishable bands } \\
1 \text { more than three distinguishable bands }\end{array}$ \\
\hline Bands distinct or indistinct & $\begin{array}{l}0 \text { bands indistinct; resembles noise } \\
1 \text { bands distinct }\end{array}$ \\
\hline Horizontal & $\begin{array}{l}0 \text { general tone or fundamental not constant in pitch } \\
1 \text { tone constant in pitch }\end{array}$ \\
\hline Chevron & $\begin{array}{l}0 \text { non-chevron-shaped } \\
1 \text { chevron-shaped }\end{array}$ \\
\hline Variability & $\begin{array}{l}0 \text { bands among figures similar in shape } \\
1 \text { bands among figures variable in shape }\end{array}$ \\
\hline Lower and upper frequency limits, bandwidth & from Table 1 \\
\hline
\end{tabular}




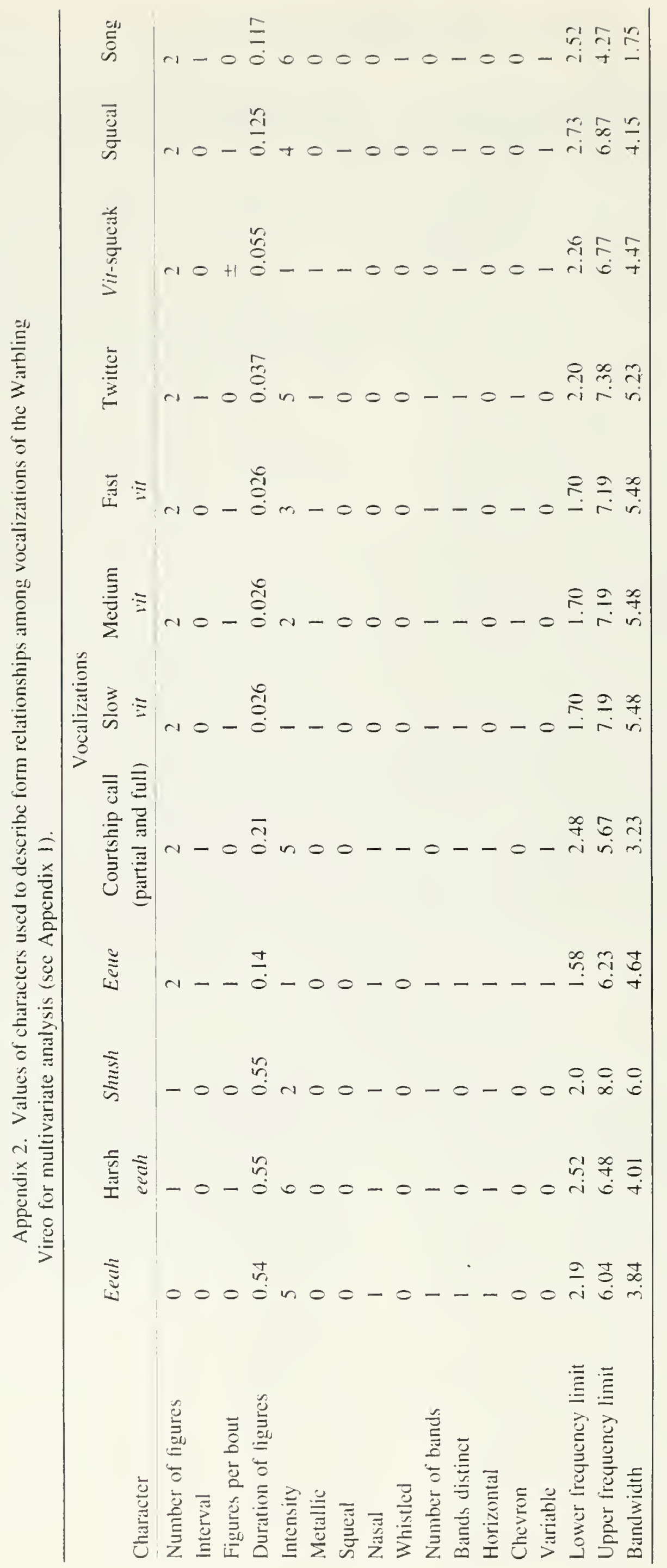




\section{Literature Cited}

ANDREW, R. J.

1957 A comparative study of the calls of Emberiza species. lbis $99: 27-42$.

1961a The motivational organization controlling mobbing calls of Blackbirds (Turdus merula), I, 11, III, IV. Behaviour 17:224-246, 288-321: 18:25-43, 161-176.

$1961 \mathrm{~b}$ The displays given by passerines in courtship and reproduction: a review. Ibis 19:549-579.

1964 Vocalizations in chicks and the concept of stimulus contrast. Animal Behaviour 12:64-76.

ARMSTRONG, E. A.

1973 A study of bird song. 2nd ed. New York, Dover. $343 \mathrm{pp}$.

BAERENDS, G. P.

1976 The functional organization of behaviour. Animal Behaviour 24:726-738.

BARLOW, G. W.

1977 Modal action patterns. In Sebeok, T. A., ed., How animals communicatc. Bloomington, Indiana University Press, pp. 98-136.

BARLOW, J. C.

1962 Natural history of the Bell Vireo (Vireo bellii, Audubon). University of Kansas Publications in Zoology 12:241-296.

1980 Patterns of ecological interactions among migrant and resident vireos on the wintering grounds. In Keast, A. and E. S. Morton, eds., Migrant birds in the Neotropics: ecology, behavior, distribution and conservation. Washington. D.C., Smithsonian Institution Press, pp. 80-107.

BARLOW, J. C. and J. C. RICE

1977 Aspects of the comparative behaviour of Red-eyed and Philadelphia vireos. Canadian Journal of Zoology $55: 528-542$.

BENT, A. C.

1950 Life histories of North American wagtails, shrikes, vireos and their allies. United States National Museum Bulletin 197:1-411.

BERTALANFFY. L. von

1950 The theory of open systems in physics and biology. Science 111:23-29.

1962 An essay on the relativity of categories. General Systems 7:71-84.

1968 General systems theory. New York, George Braziller. 289 pp.

1975 Perspectives on general systems theory. New York, George Braziller. 183 pp.

BROCKWAY, B. F.

1969 Role of Budgerigar vocalizations in the integration of breeding behaviour. In Hinde, R. A., ed., Bird vocalizations. Cambridge, Cambridge University Press, pp. $131-158$.

CARYL. P.

1975 Sexual behaviour in the Zebra Finch (Taeniopygia guttata): response to familiar and novel partners. Animal Behaviour 24:93-107.
CATCHPOLE, C. K

1982 The cvolution of bird sounds in relation to mating and spacing bchavior. In Kroodsma, D. E. and E. H. Miller, eds., Acoustic communication in birds. New York, Academic Press, vol. I, pp. 297-319.

CAVALLO, R. E.

1979 Systems research movement: characteristics, accomplishments, and current developments. General Systems Bulletin 9:1-131.

COHEN, S. M., W. C. STEBBINS, and D. B. MOODY

1978 Audibility thresholds of the Bluc Jay. Auk 95:563568.

COLLIAS, N. E. and M. Joos

1953 The spectrographic analysis of sound signals of the domestic fowl. Behaviour 5:175-188.

CURIO, E.

1975 The functional organization of anti-predator behaviour in the Pied Flycatcher, a study of avian visual perception. Animal Behaviour 23:1-115.

DAWKINS, R, and J. R. KREBS

1978 Animal signals: information or manipulation? In Krebs, J. R. and N. B. Davies, eds., Behavioural ecology. Oxford, Blackwell, pp. 282-309.

DOOLING, R. J.

1982 Auditory perception in birds. In Kroodsma, D. E. and E. H. Miller. eds., Acoustic communication in birds. New York, Academic Press, vol. 1, pp. 95-131.

ERICKSON, C. J.

1973 Mate familiarity and reproductive behavior of Ringed Turtle Doves. Auk 90:780-795.

FENTRESS, J. C.

1973 Specific and nonspecific factors in the causation of behavior. In Bateson. P. P. G. and P. H. Klopfer. eds., Perspectives in ethology. New York, Plenum Press, pp. 155-224.

1976 Dynamic boundaries of patterned behaviour: interaction and self-organization. In Bateson, P. P. G. and R. A. Hinde, eds., Growing points in ethology. Cambridge, Cambridge University Press, pp. 135-170.

FICKEN, M. S., R. W. FICKEN, and S. R. WITKIN

1978 Vocal repertoire of the Black-capped Chickadee. Auk 95:34-48.

GODFREY, W. E

1986 Birds of Canada. National Museum of Natural Seiences. 596 pp.

GREENEWALT, C. $\mathrm{H}$.

1968 Bird song: acoustics and physiology. Washington, Smithsonian Institution Press. 194 pp.

HALL, A. D. and R. E. FAGEN

1975 Definition of system. In Rubin, B. R. and J. Y. Kim, eds., General systems theory and human communication. Rochelle Park, N.J., Hayden, pp. 52-65.

HARARY, F. and M. F. BATI:LL.

1981 What is a system? General Systems 26:61-72.

HARDY, J. W

1979 Vocal repertoire and its possible evolution in the black 
and blue jays (Cissilopha). Wilson Bulletin 91:187201.

IIARTSHORNI:, C.

1956 The monotony-threshold in singing birds. Auk 73:176192.

1973 Born to sing: an interpretation and world survey of bird song. Bloomington, Indiana University Press. $304 \mathrm{pp}$.

HELLIGINHERG, W.

1974 Processes governing behavioural states of readiness Advances in the Study of Behaviour 5:175-200.

HINDE, R. A.

1955/ A comparative study of the courtship of certain finches 1956 (Fringillidae). Ibis 97:706-745; 98:1-23.

1965 Interaction of internal and external factors in the integration of canary reproduction. In Beach. F. A., ed., Sex and behavior. New York, Wiley and Sons, pp. 381-415.

1969 Bird vocalizations. Cambridge, Cambridge University Press. 394 pp.

HOWES-JONES, D.

1982 The structure of an avian communication system. Ph.D. thesis, University of Toronto. 374 pp.

1984 The vocal behaviour of young Warbling Vireos. Canadian Journal of Zoology 62:1714-1719.

1985a Relationships among song activity, context and social behavior in the Warbling Vireo. Wilson Bulletin 97: 4-20.

1985b Activity patterns and nesting habits of Warbling Vireos in southern Ontario. Canadian Field-Naturalist 99:484-489.

1985c The complex song of the Warbling Vireo. Canadian Journal of Zoology 63:2756-2766.

HUXLEY, C. R. and R. WILKINSON

1977 Vocalizations of the Aldabra White-throated Rail Dryolimnas cuvieri aldabramus. Proceedings of the Royal Society of London (B) 197:315-331.

HUXLEY, J.

1923 Courtship activities of the Red-throated Diver ( $\mathrm{Co}$ lymbus stellatus Pontopp.). with a discussion of the evolution of courtship in birds. Journal of the Linnean Society of London 35:253-292.

ISAAC, D. and P. MARLER

1963 Ordering of sequences of singing behaviour of Mistle Thrushes in relation to timing. Animal Behaviour 11:179-188.

JENNI, D. A., R. D. GAMBIS, and B. J. BETTS

1974 Acoustic behavior of the Northern Jacana. Living Bird 13:193-210

JOIINSTON, T. D

1976 Theoretical considerations in the adaptation of animal communication systems. Journal of Theoretical Biology 57:43-72.

KOESTLER, A. and J. R. SNYTHHES

1969 Beyond reductionism. London, Hutchison. 438 pp. KONISHII, N

1963 The role of auditory feedback in the vocal behaviour of the domestic fowl. Zeitschrift für Tierpsychologie $20: 349-367$
KREBS, J.R.

1977 The significance of song repertoires: the Beau Geste hypothesis. Animal Behaviour 25:475-478.

KRIPPIENDORF, K.

1975 The systems approach to communication. In Rubin, B. R. and J. Y. Kim, eds., General systems theory and human communication. Rochelle Park. N.J., Hayden, pp. 138-163.

KROOLSMA. D. E.

1977 Correlates of song organization among North American wrens. American Naturalist 111:995-1008.

1978 Continuity and versatility in bird song: support for the monotony threshold hypothesis. Nature 274:681-683.

KROODSMA, D. E. and E. H. MILLER

1982 Acoustic communication in birds: production, perception, and design features of sound. New York. Academic Press, vol. 1. 371 pp.

LANYON, W. E.

1960 The ontogeny of vocalizations in birds. In Lanyon, W. E. and W. N. Tavolga, eds., Animal sounds and communication. American Institute of Biological Sciences Publication 7. Washington. D.C. The Institute, pp. $321-348$.

LASZLO, E.

1972 Introduction to systems philosophy. New York. Harper and Row. 328 pp.

1975 Basic constructs of systems philosophy. In Rubin, B. R. and J. Y. Kim, eds., General systems theory and human communication. Rochelle Park. N.J.. Hayden. pp. 66-78.

LAWRENCE. L. de K.

1953 Nesting life and behaviour of the Red-eyed Vireo. Canadian Field-Naturalisı 67:47-87.

LEHRMAN, D. S.

1965 Interaction between internal and external environment in the regulation of the reproductive cycle of the Ring Dove. In Beach. F. A., ed., Sex and behavior. New York. Wiley and Sons, pp. 355-380.

LEIN, M. R.

1980 Display behavior of Ovenbirds (Seiurus aurocapillus) 1. Non-song vocalizations. Wilson Bulletin 92:312329.

LENION, R. E.

1968 The relation between organization and function of song in Cardinals. Behaviour 32:158-178.

1971 Analysis of song of Red-eyed Vireos. Canadian Journal of Zoology 49:847-854.

MARLER, P.

1955 Characteristics of some animal calls. Nature 176:6-8.

1956 The voice of the Chaffinch and its function as a language. Ibis 98:231-261.

1961 The logical analysis of animal communications. Journal of Theoretical Biology 1:295-317.

1965 Communication in monkeys and apes. In DeVore. 1.. ed., Primate behavior: field studies of monkeys and apes. New York. Holt, Rinehart and Winston. pp. $544-584$.

1967 Animal communication signals. Science 157:769-774.

1968 Aggregation and dispersal: two functions in primate 
communication. In Jay, P., ed., Prinates: studics in adaptation and variability. New York, Holt, Rinchart and Winston. pp. 428-438.

1969 Tonal quality of bird sounds. In Hinde, R. A.. ed.. Bird vocalizations. Cambridgc. Cambridge University Press. pp. 5-18.

1976a Social organization, communication, and graded signals in the chimpanzee and the gorilla. In Bateson, P. P. G. and R. A. Hinde, eds., Growing points in ethology. Cambridge, Cambridge University Press, pp. 239-281.

1976b On animal aggression: the roles of strangeness and familiarity. American Psychologist 31:239-246.

MARTEN, K. and P. MARLER

1977 Sound transmission and its significance for animal communication 1: temperate habitats. Behavioral Ecology and Sociobiology 2:271-290.

MCFARLAND. D. J. and R. SIBLEY

1975 The behavioural final common path. Philosophical Transactions of the Royal Society of London (B) 270:265-293.

NILLER, J. G.

1978 Living systems. New York, McGraw-Hill. 1102 pp. MOLES, A.

1963 Animal language and information thcory. In Busnel, R. G.. ed.. Acoustic behaviour of animals. Amsterdam. Elsevier, pp. 121-131.

MORRIS. D.

1957 'Typical intensity' and its relation to the problem of ritualization. Behaviour 11:1-12.

MORTON, E. S

1975 Ecological sources of selection on avian sounds. American Naturalist 109:17-34.

1977 On the occurrence and significance of motivationalstructural rules in some bird and mammal sounds. American Naturalist 111:855-869.

1982 Grading, discreteness, redundancy, and motivationalstructural rules. In Kroodsma, D. E. and E. H. Miller. cds., Acoustic communication in birds. New York, Academic Press, vol. 1. pp. 183-213.

MOYNIHAN, M. H

1970 Control, suppression. decay, disappcarance, and replacement of displays. Journal of Theoretical Biology 29:85-112.

NOTTEBOHM. F.

1975 Continental patterns of song variability in Zonotrichia capensis: some ecological correlates. American Naturalist 109:605-624.

ORING, L. W'

1968 Vocalizations of the Green and Solitary sandpipers. Wilson Bullctin 80:395-420.

PIAGET. J.

1970 Structuralism. New York. Harper and Row. $153 \mathrm{pp}$.

1971 Biology and knowledge. Chicago, Chicago University Press. 384 pp.

RAPOPORT, A.

1975 Modern systems theory - an outlook for coping with change. In Rubin, B. D. and J. Y. Kim, eds., General systems theory and human communication. Rochelle
Park, N.J., Hayden, pp. 33-51.

1978 What is semantics? General Systems 23:159-164.

RICE, J. C.

1974 Social and competitive interactions between two species of vireos (Aves: Vireonidae). Ph.D. thesis, University of Toronto. $292 \mathrm{pp}$.

SEBEOK. T. A

1965 Animal communication. Science 147:1006-1014. SCHLEIDT, W. $M$.

1973 Tonic communication: continual effects of discretc signs in animal communication systcms. Journal of Theoretical Biology 42:359-386.

SHIOVITZ, K. A.

1976 The process of species-specific recognition by the Indigo Bunting (Passerina cyanea) and its relationship to the organization of avian acoustic behaviour. Behaviour 55:129-175.

SILCOX, A. P. and S. M. EVANS

1982 Factors affecting the formation and maintenance of pair bonds in the Zebra Finch (Taeniopygia guttata). Animal Behaviour 30:1237-1243.

SMITH, W. J.

1966 Communication and relationships in the genus Tyramus. Nuttall Ornithological Club Publication $6: 1-250$.

1969 Messages of vertebrate communication. Science 165 145-150.

1970a Song-like displays in the genus Savornis. Behaviour $37: 64-84$.

1970b Displays and message assortment in Sayornis species. Behaviour 37:85-113.

1977 The behaviour of communicating. Cambridge. Cambridge University Press. $545 \mathrm{pp}$.

SMITH, W. J., J. PAWLUKIEWICZ, and S. T. SMITH

1978 Kinds of activities corrclated with singing patterns of the Yellow-throated Virco. Animal Behaviour 26:862884.

SNEATH, P. H. A and R. R. SOKAL

1973 Numerical taxonomy. San Francisco, W. H. Frecman. $359 \mathrm{pp}$

SOKAL, R. R. and F. J. ROHLF

1969 Biometry. San Francisco, W. H. Freeman. 776 pp. SUTHERLAND, J. W

1973 A general systems philosophy for the social and behavioral sciences. New York, George Braziller. 202 pp.

THOMPSON, W. L.

1972 Singing behaviour of the Indigo Bunting (Passerina cyanea). Zcitschrift für Ticrpsychologic 31:39-59.

1976 Vocalizations of the Lazuli Bunting. Condor 78:195207.

THOMPSON, W. L. and J, O. RICE

1970 Calls of the Indigo Bunting (Passerina ryanea). Zeitschrift für Tierpsychologie 27:35-46.

THORPE. W. H. and J. HAL.L-CRAGGS

1976 Sound production and perception in birds as related to the general principles of patter perception. In Bateson. P. P. G. and R. A. Hinde, eds., Growing points in ethology. Cambridge. Cambridge University Press. 
pp. $171-190$.

TINIHI:RGEN. N.

1951 The study of instinct. Oxford, Oxford University Press. $228 \mathrm{pp}$.

1952 Derived activities: their causation, biological significance, origin and emancipation during evolution. Quarterly Review of Biology 27:1-32.

WILIEY, R. H.

1975 Multidimensional variation in an avian display: implications for social communication. Science 190:482483.

1976a Communication and spatial relationships in a colony of Common Grackles. Animal Behavjour 24:570-584.

1976b Affiliation between sexes in Common Grackles. 1. Specificity and seasonal progression. Zeitschrift für Ticrpsychologic 40:59-79.

1976c Affiliation between sexes in Common Grackles. 11. Spatial and vocal coordination. Zeitschrift für Tier- psychologic 40:244-264.

WII.KINSON. R. and P. E. HOWSE.

1975 Variation and temporal characteristics of the vocaliZations of Bullinches. Zcitschrift für Tierpsychologje 38:190-211.

WILSON, E. O.

1971 The insect socicties. Cambridge. Massachusetts. Belknap Press of Harvard University Press. 548 pp.

1975 Sociobiology. Cambridgc. Harvard University Press. 697 pp.

ZANN, R.

1975 Inter-and intraspecific variation in the calls of three species of grassfinches of the subgenus Poephila (Gould) (Estrildidae). Zeitschrift für Tierpsychologic $39: 85-125$.

ZIPF, G. II.

1935 Psychobiology of language. Boston. Houghton Mifflin. $336 \mathrm{pp}$. 
0 

